\title{
Nighttime Pc3 pulsations: MM100 and MAGDAS observations
}

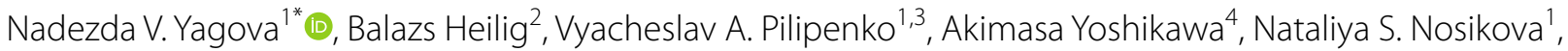 \\ Kiyohumi Yumoto ${ }^{4}$ and Jan Reda ${ }^{5}$
}

\begin{abstract}
In this paper, we present a statistical and case analysis of nighttime Pc3 pulsations observed from middle to equatorial latitudes during the year 2003. We found two groups of nighttime Pc3 pulsations. Pc3s of the first group are in fact the nightside counterpart of morning Pc3 pulsations with large azimuthal scales slowly attenuating toward midnight. Such night signatures of morning Pc3 waves are observed during the periods of fast solar wind $(V>500 \mathrm{~km} / \mathrm{s})$. The second type is the locally generated night Pc3 pulsations. They can be observed under moderate solar wind velocities. Maximal occurrence rates and amplitudes for these pulsations are recorded at middle geomagnetic latitudes near the local magnetic midnight. Probably, they are associated with auroral activations or local non-substorm bursty processes.
\end{abstract}

Keywords: Geomagnetic pulsations, lonosphere, Magnetosphere

\section{Background}

Narrowband Pc3 geomagnetic pulsations in the frequency band $20-80 \mathrm{mHz}$ are observed regularly at middle latitudes almost every day. The midlatitude Pc3 pulsations are commonly recorded in a wide range of magnetic local times (MLT) from early morning to late evening hours, whereas the maximal amplitudes and occurrence rates are observed in the morning/pre-noon MLT sector. These pulsations are considered as a ground image of upstream waves generated in the terrestrial foreshock. The frequency of upstream waves is determined by the magnitude $B_{\text {IMF }}$ of the interplanetary magnetic field (IMF) (Guglielmi and Troitskaya 1974) due to their ion-cyclotron origin. Following Villante and Tiberi (2016), we use the $f-B_{\text {IMF }}$ dependence in its simplest form, suggested in Troitskaya (1988)

$$
f[m H z]=6 B[n T]
$$

However, the relationship (1) has a statistical character and holds for values averaged over a long (e.g., hourly) time interval. On the wave event basis, this

\footnotetext{
*Correspondence: nyagova@yandex.ru

${ }^{1}$ Institute of Physics of the Earth, Moscow, Russia

Full list of author information is available at the end of the article
}

correspondence may become rather uncertain because of a highly structured solar wind and IMF. The accuracy of the OMNI data set in characterizing conditions near the nose of Earth's bow shock under predominantly radial IMF is not better than $80 \%$ (Bier et al. 2014).

The generation of upstream waves is most efficient in the region of the quasi-parallel bow shock, where angles between the IMF and the normal to the bow shock are small (Guglielmi and Troitskaya 1974; Greenstadt and Olson 1976; Heilig et al. 2010; Lotz et al. 2015). In earlier studies, Kelvin-Helmholtz instability (KHI) at the flanks of the magnetopause was often considered as a possible mechanism of Pc3-5 wave generation, resulting in morning and evening maxima in diurnal variation of Pc3 occurrence rate and in the growth of Pc3 amplitudes with the solar wind speed (Singer et al. 1977; Greenstadt et al. 1979). Further, wave energy is transported from the magnetopause across the dayside magnetosphere toward the ionosphere as a fast magnetosonic mode (Yumoto et al. 1985). Upon this propagation, a part of the wave energy is resonantly converted into Alfven standing waves and cavity oscillations. Such cavity can be formed in a region with the minimum of Alfven velocity, e.g., inside the plasmasphere dome (Gul'elmi 1970). Therefore, spectra of ground signals are formed as a combination of 
the upstream wave spectra and resonant response of the Alfven resonator between the conjugate ionospheres or in magnetospheric cavities (Vellante et al. 1995; Vero et al. 1998; Pilipenko et al. 1999).

Pc3 waves are sensitive to the properties of the ionosphere. Fast mode is weakly absorbed in the dayside ionosphere and does not "feel" the nightside ionosphere. Absorption of Alfven waves depends on the contrast between the ionospheric height-integrated Pedersen conductance $\Sigma_{P}$ and wave conductance $\Sigma_{A}$ of the upper ionosphere. According to this notion, Pc3 pulsation absorption in the ionosphere is to be low at dayside, where $\Sigma_{P} \gg \Sigma_{A}$, but must increase significantly at nightside, where $\Sigma_{P} \leq \Sigma_{A}$ Pilipenko et al. (2008). In the region of strong lateral gradient of the ionospheric conductance (dawn terminator) Pc3 waves experience a change in the polarization ellipse orientation (Saka and Alperovich 1998) and a phase jump between longitudinally separated stations (Waters et al. 2001).

Generally, Pc3 waves are believed to be a dayside phenomenon, though their occurrence at nighttime has been noticed in several case studies. Recording of Pc3-4 pulsation activity on a chain of magnetometers aligned east-west at middle geomagnetic latitudes showed no evidence of the anti-sunward propagation, predicted by the KHI mechanism (Mier-Jedrzejowicz and Southwood 1979). At the same time, highly coherent signals occurred near midnight, so the nightside was proposed to be the source region for a significant part of the Pc3-4 pulsation activity. Coherent variations of Pc3-4 power in the proximity of the dayside cusp and at the nightside plasmapause were reported by Ponomarenko and Waters (2010). These variations followed the variations of the IMF magnetic field magnitude and cone angle, indicating the upstream wave origin of the observed nighttime wave event. Compressional mode Pc3 waves were detected simultaneously at low latitudes at nightside by low-orbiting satellite and ground stations (Cuturrufo et al. 2015). Thus, these case studies indicated that dayside and nightside Pc3-4 waves are coupled.

However, the dominating type of ULF waves at nighttime is the transient Pi2 pulsations (Sutcliffe and Lyons 2002; Kim et al. 2005; Keiling and Takahashi 2011). Although auroral substorm disturbance is confined in a relatively narrow range of longitudes around local midnight, Pi2s at low latitudes can be observed over a very large range of longitudes (Yumoto 2001). These results have suggested a global cavity mode as a mechanism responsible for the spatial structure of low-latitude Pi2 pulsations. Typical period of low-latitude Pi2 pulsations, from a fraction of a minute to few minutes, is believed to be determined either by eigenperiod of the plasmasphere cavity mode (Sutcliffe et al. 2013) or by a forced response to a periodic energy release in the magnetotail (Keiling and Takahashi 2011). Takahashi et al. (2005) analyzed nightside Pc4s and Pi2s and found that these two types of ULF waves were generated by different physical mechanisms, although their central frequencies were close.

A comprehensive statistical analysis of nighttime Pc3 waves was performed with the data of L'Aquila observatory $(L=1.6)$ (Villante and Tiberi 2016). The authors found that nighttime Pc3s had lower amplitudes than those at dayside, but their principal features, such as the IMF control of the central frequency, were the same. Similarly to dayside pulsations, one of the spectral peaks seen in both horizontal components was associated with the upstream wave frequency (1), while another peak seen mostly in the north-south component was interpreted as a signature of the local field line resonance. The authors grouped nighttime Pc3-4 waves into three frequency ranges and found that intermediate- and high-frequency events demonstrated typical Pc3 features, while regularities of low-frequency events were alike those known for Pi2 pulsations.

In this paper, we analyze in a greater detail nighttime Pc3 pulsations from middle to equatorial latitudes using the data from two meridional networks in Europe and in the Pacific region, augmented with some equatorial stations. We analyze spatial distributions and spectral parameters of individual nighttime Pc3 events and their statistical properties using the data of the whole year 2003. The choice of the observational period is determined by the best data coverage at both magnetometer networks and by a relatively high level of solar and geomagnetic activity after the maximum of the 23 solar cycle.

\section{Observational data and their processing}

MAGDAS (MAGnetic Data Acquisition System) is a global magnetometer network covering geomagnetic latitudes from auroral to equatorial in the Pacific region (Yumoto 2006). MAGDAS includes an additional chain of stations along the geomagnetic dip equator. The MAGDAS stations are equipped with three-component fluxgate magnetometers (3-s sampling period). For our analysis, we have used the data from Eastern Asia, Oceania, and Australia forming three meridional chains along geomagnetic longitudes $\Lambda \approx 185^{\circ}, \Lambda \approx 210^{\circ}$, and $\Lambda \approx 225^{\circ}$, covering geomagnetic latitudes from auroral zone to the equator.

The European magnetometer MM100 network (Heilig et al. 2007) ranges from auroral to middle latitudes along the geomagnetic longitude $\Lambda \approx 100^{\circ}$. The MM100 stations are equipped with fluxgate magnetometers with sampling rate $1 \mathrm{~Hz}$ or higher. From this array, we have selected three stations with the lowest levels of industrial interference. 
The locations of the selected stations are shown in Fig. 1, and their coordinates are given in Table 1. The data used for the analysis were recorded during the year 2003 . The data coverage is the best for the first four months of the year. We use this interval to formulate the hypothesis and then verify it on the data of the whole year. Besides, some of the results on Pc3 spatial distribution are obtained on the four-month data set, while the others (like different space weather conditions for two types of pulsations) are based on the data of the whole year (see "Statistical relationships section" for details).

The data were transformed into the system aligned along the corrected geomagnetic (CGM) coordinates. The 1-s data from the MM100 stations have been low-passed-filtered with the cutoff frequency $f=$ $166 \mathrm{mHz}$ and then resampled to the common cadence $3 \mathrm{~s}$. All the data have been high-pass-filtered with a cutoff frequency $10 \mathrm{mHz}$. The power spectral density (PSD) $P_{f}$ for each horizontal component has been calculated with the Blackman-Tukey method (Jenkins and Watts 1968; Kay 1988). The spectral coherence $\gamma^{2}$ has been estimated for all the station pairs. Parameters of the spectral estimate are as follows: length of the time window $N=256$ samples $(768 \mathrm{~s})$, length of the Kaiser window $m=32$ samples. Variance of PSD auto-spectra $S_{X X}$ and $S_{Y Y}$ and cross spectrum $S_{X Y}$ is $\operatorname{Var}(S) / S=2 m / 3 N=1 / 12$ (Kay 1988). Spectral coherence $\gamma^{2}$ and its variance are calculated as (Jenkins and Watts 1968)

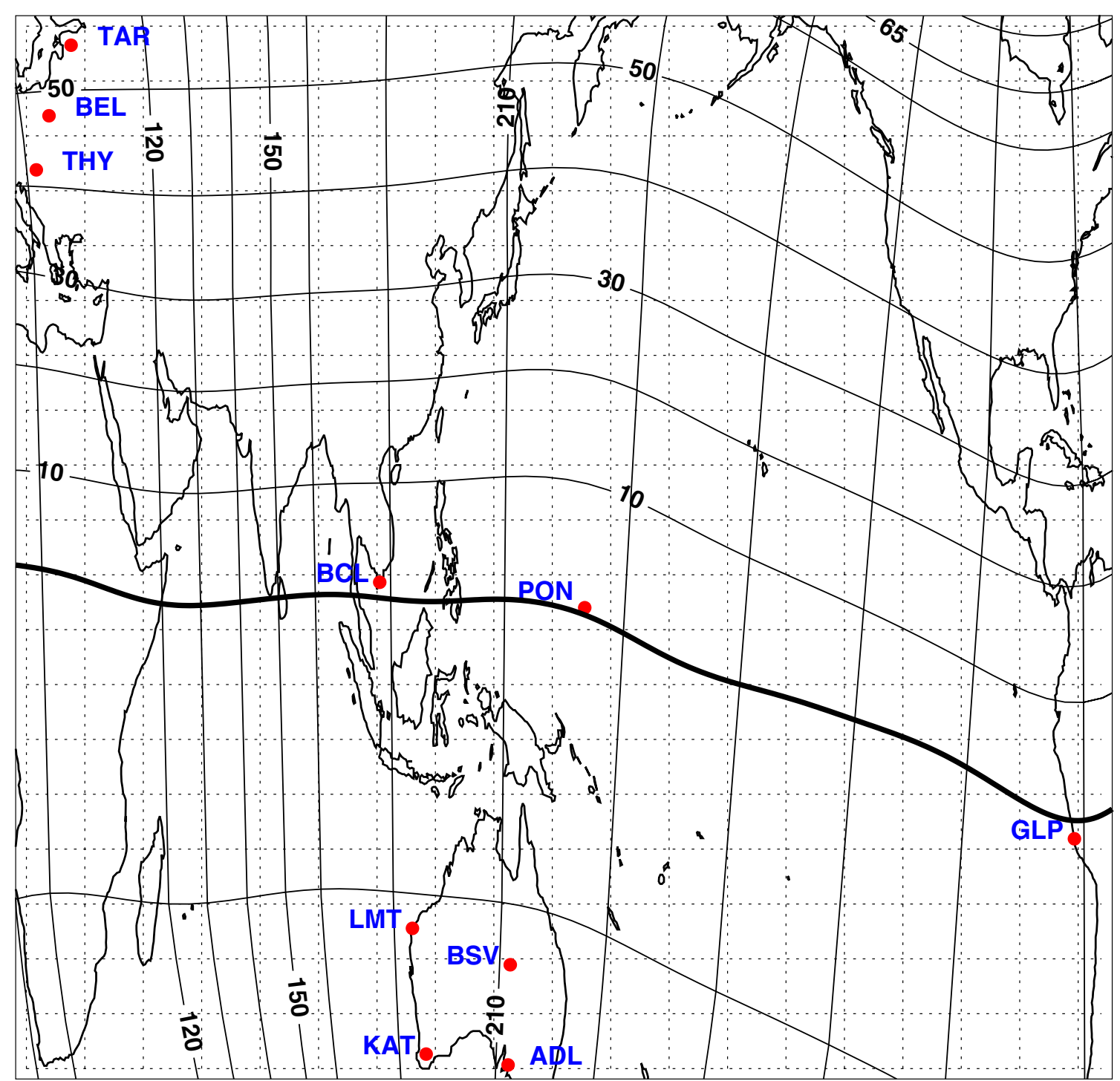

Fig. 1 A map with magnetic stations used in the study. Dip equator is shown as a bold line. Thin solid lines denote geomagnetic coordinates 
Table 1 Coordinates of stations

\begin{tabular}{|c|c|c|c|c|c|c|c|}
\hline \multirow[t]{2}{*}{ Station } & \multirow[t]{2}{*}{ Network } & \multicolumn{2}{|c|}{ Geographic } & \multicolumn{2}{|l|}{ CGM } & \multirow[t]{2}{*}{$L$} & \multirow{2}{*}{$\begin{array}{l}\text { MLT } \\
\text { midnight }\end{array}$} \\
\hline & & LAT & LON & $\Phi$ & $\boldsymbol{\Lambda}$ & & \\
\hline BEL & MM100 & 51.83 & 20.80 & 48.01 & 96.15 & 2.23 & $21: 49$ \\
\hline TAR & MM100 & 58.26 & 26.46 & 54.46 & 103.07 & 3.01 & $21: 21$ \\
\hline THY & MM100 & 46.90 & 17.54 & 41.92 & 92.01 & 1.83 & $22: 12$ \\
\hline$A D L$ & MAGDAS & -34.67 & 138.65 & -45.95 & 213.96 & 2.10 & $14: 22$ \\
\hline $\mathrm{BCL}$ & MAGDAS & 9.32 & 105.71 & 0.70 & 177.12 & 1.00 & 17:04 \\
\hline BSV & MAGDAS & -25.54 & 139.21 & -35.78 & 213.24 & 1.54 & $14: 22$ \\
\hline GLP & MAGDAS & -14.06 & 284.05 & -0.06 & 355.57 & 1.00 & $04: 47$ \\
\hline KAT & MAGDAS & -33.68 & 117.62 & -46.14 & 188.75 & 2.12 & $16: 26$ \\
\hline LMT & MAGDAS & -22.22 & 114.10 & -33.30 & 185.51 & 1.45 & $16: 44$ \\
\hline PON & MAGDAS & 7.00 & 158.33 & 0.08 & 229.19 & 1.00 & $13: 51$ \\
\hline
\end{tabular}

$$
\gamma^{2}=\frac{S_{X Y}^{2}}{S_{X X} S_{Y Y}} \quad \operatorname{Var}\left(\gamma^{2}\right)=\frac{2 \gamma^{2}\left(1-\gamma^{2}\right)^{2}}{N}
$$

The variance does not depend on window width $m$ and becomes small in spectral domain where $\gamma \rightarrow 1$.

The program developed for the automatic selection of intervals with significant Pc3 activity is based on the spectrum analysis algorithm described in Yagova et al. (2015) and the quantitative characterization of ULF spectra developed in Yagova et al. (2010). Typically, a PSD in the Pc3 frequency range decreases with frequency as $P_{f}(f) \propto f^{-\alpha}$, that corresponds to colored (red) noise. We use a $\log$-log spectrum $\Pi(F)$, where $\Pi=\log \left(P_{f}\right)$, and $F=\log (f)$. For colored noise, the dependence $\Pi_{c n}(F)$ is linear $\Pi_{c n}=a_{0}-a_{1} F$ ( $a_{0}$ and $a_{1}$ are constants). To reveal spectral maxima associated with a harmonic signal, the function $\Pi(F)$ is linearly detrended ("whitened") as $\Pi_{w}(F)=\Pi(F)-\Pi_{c n}(F)$ (here $\Pi_{c n}$ is the best linear fit of $\Pi(F)$ in the interval $\left[F_{L}, F_{R}\right]$, where $F_{L, R}=\log \left(f_{L, R}\right)$, and $f_{L, R}$ are the boundaries of frequency interval). In a $\Delta F$ vicinity of $F=F_{\max }$ where $\Pi_{w}$ reaches a maximum, $\Pi_{w}(F)$ is approximated by a square polynomial $\Pi_{w}=-a_{2}\left(F-F_{\max }\right)^{2}+\Pi_{w 0}$. A time interval is selected as a Pc3 interval if both $P\left(f_{\max }\right)$, where $F_{\max }=\log \left(f_{\max }\right)$, and $a_{2}$ exceed some threshold values $P_{b}$ and $a_{b}$. This scheme is illustrated in Fig. 2.

The procedure of automatic detection is organized as an alternative to the visual selection of Pc3 events. Detection parameters are selected in the following way. At the first stage, Pc3 events are selected visually. The values of $P_{b}$ and $a_{b}$ are taken in accordance with their distribution in the intervals which are visually selected. Then agreement of visible and automatic selection results are checked for a longer time interval and parameters are corrected. After several iterations, the parameters for automatic detection are fixed and applied to

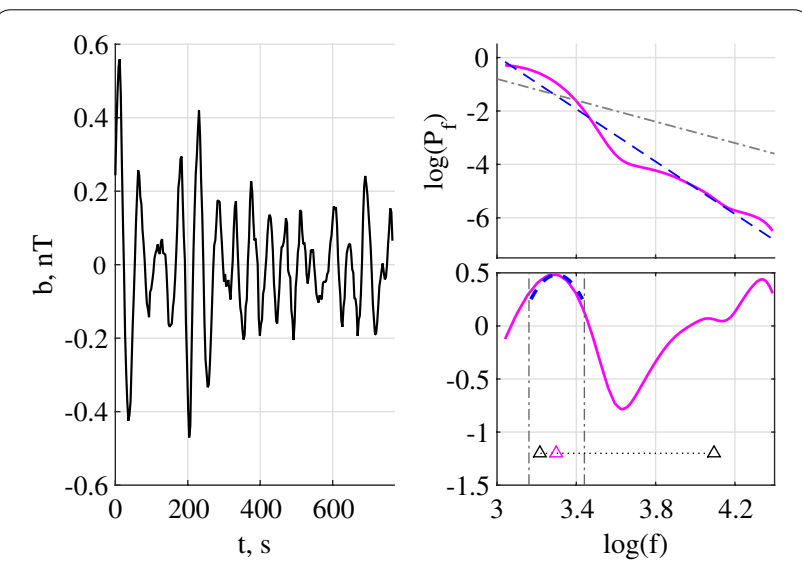

Fig. 2 An illustration of the Pc selection method. High-pass-filtered magnetogram is shown at left panel, log-log PSD spectrum $\Pi(F)$ and its linear approximation is given at right upper panel. Log-log spectrum and its linear approximation are shown in the upper right panel with solid magenta and dashed blue lines, respectively. A dash-dotted gray line at the upper right panel shows the lower boundary of maximal PSD logarithm $\Pi(F)$ at $F=F_{\text {max }}$. A linear detrended (whitened) log-log spectrum $\Pi_{w}(F)$ is shown at the right lower panel (magenta) and its parabolic approximation in the vicinity of maximum is shown with a blue dashed line. Boundaries of allowed $F$ interval for a maximum of $\Pi_{w}$ are shown with black triangles, and the magenta triangle shows the frequency logarithm $F_{\max }$ of the actual $P i_{W}(F)$ maximum

the entire data set. In the present study, we use the parameters $f_{L}=25 \mathrm{mHz}, f_{R}=60 \mathrm{mHz}, P_{b}=0.3\left(f / f_{L}\right)^{2}$, $a_{b}=0.33 f_{\max }$, and $\Delta F=0.15$. The selected parameter set corresponds to threshold amplitudes about $0.2 \mathrm{nT}$ at $30 \mathrm{mHz}$.

\section{Examples of typical nighttime Pc3 events}

First, we present several examples of nighttime Pc3 pulsations to show their typical waveforms and spectral features. All spectral maxima $f_{c}$ found at two or more 
stations are checked for the correspondence (1) with the IMF-controlled Pc3 frequency $f_{B}$.

\section{Pc3 wave event at nighttime on DOY $=23$}

Pc3 pulsations with quasi-period of about $40 \mathrm{~s}$ were recorded at two magnetic meridians MM185 and MM210 on $\mathrm{DOY}=23,2003$ (onset 17:21 UT). During the time interval analyzed, all the stations were in the night sector $(\mathrm{MLT} \approx 1$ for MM185 and MLT $\approx 3$ for MM210). Highpass-filtered magnetograms for both horizontal components are shown in Fig. 3 for three stations along each meridional chain starting from the equator (upper panel) up to $L=2.1$ (bottom panel).

PSD spectra are shown in Fig. 4. A spectral maximum at $f_{c 1} \approx 25 \mathrm{mHz}$ is seen at all stations, and an additional weaker maximum appears at several stations at $f_{c 2} \approx 50 \mathrm{mHz}$. At $L \approx 2.1$ stations (KAT, ADL) and at $L \approx 1.5$ stations (LMT, BSV) PSDs of $\mathrm{H}$ and D components are comparable while at equatorial stations (BCL, PON) the PSD of the D component almost vanishes. During the interval, IMF magnitude was $B=8.4 \mathrm{nT}$, and the expected frequency $f_{B}=50.4 \mathrm{mHz}$ is in good agreement with the observed frequency of the second maximum.

Coherence spectra are shown in Fig. 5. Both H and D components demonstrate highly coherent $\left(\gamma^{2}>0.5\right)$ oscillations at the frequency of the main spectral maximum at all the station pairs where pulsation is recorded.

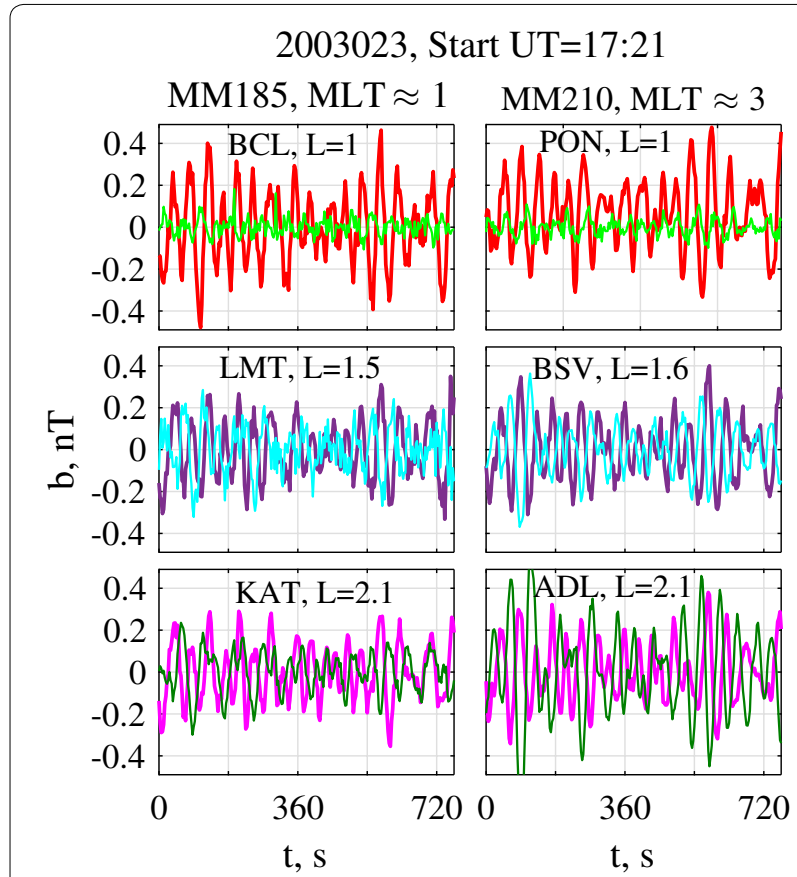

Fig. 3 Magnetograms of the $\mathrm{H}$ (bold lines) and $\mathrm{D}$ (thin lines) components along MM180 (MLT $\approx 1$, left) and MM210 (MLT $\approx 3$, right) profiles for the 768 s interval started at UT $=17: 21$ on DOY $=23$ of 2003

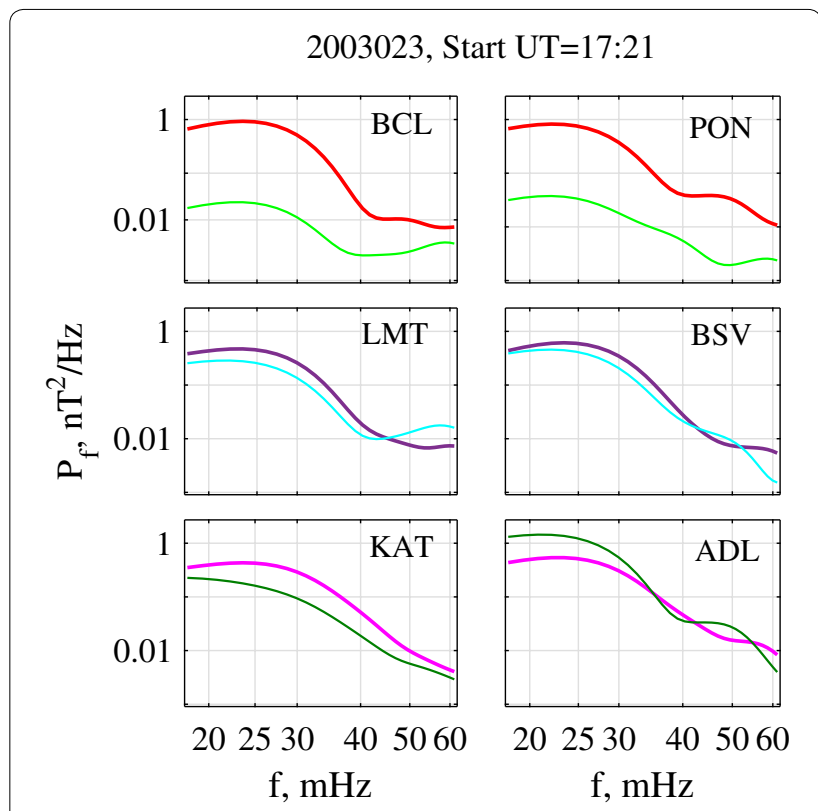

Fig. 4 PSD $P_{f}$ for the event shown in Fig. 3. H/D components are shown with bold/thin lines

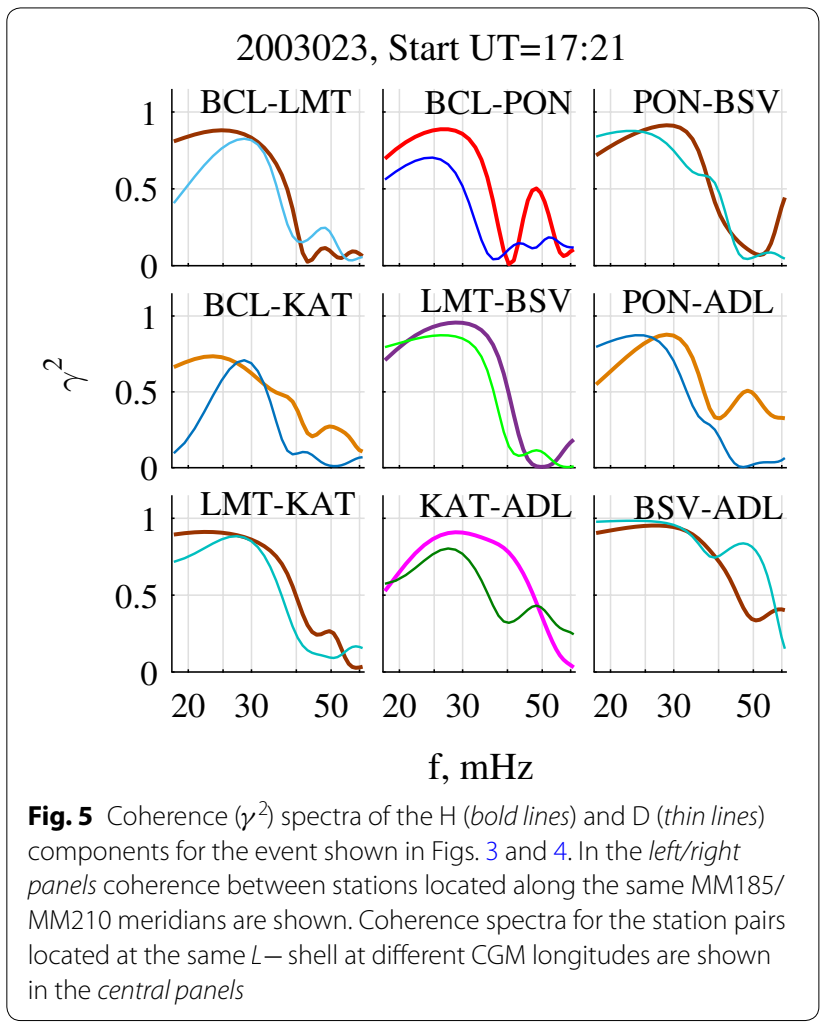

Coherence is somewhat lower only for the D component for the station pairs including equatorial stations, where the pulsation amplitudes are low. The coherence is high 
both in meridional (left and right panels) and in azimuthal (central panels) directions.

\section{Global Pc3 event on DOY 52}

Another Pc3 pulsation event was recorded on the DOY 52 of 2003 when MM100 stations were in the post-midnight sector, and MM185 and MM210 stations were in the morning-pre-noon sector. Pc3 pulsations with period of about $30 \mathrm{~s}$ are seen both in the morning and in the night sectors (Fig. 6). Simultaneous activation occurs at $t \approx 200 \mathrm{~s}$ after time interval onset (UT $=1: 33$ ) at MM100 as well as at MM185/MM210 stations. Pulsation amplitudes are higher in the morning than in the night sector and at middle $(L>2)$ than at low $(L<2)$ geomagnetic latitudes. Amplitudes of $\mathrm{H}$ and $\mathrm{D}$ components are comparable.

For this event, PSD is maximal at KAT station located in the morning sector (MLT $\approx 9$ ) at $L \approx 2.1$ (Fig. 7). Probably, at this latitude the Pc3 amplification results from local field line resonance, since only at this station the PSD of $\mathrm{H}$ component exceeds that of D component by almost an order of magnitude. For all the other locations, PSD of both horizontal components is comparable, and morning PSD is 2-3 times higher than night PSD at the

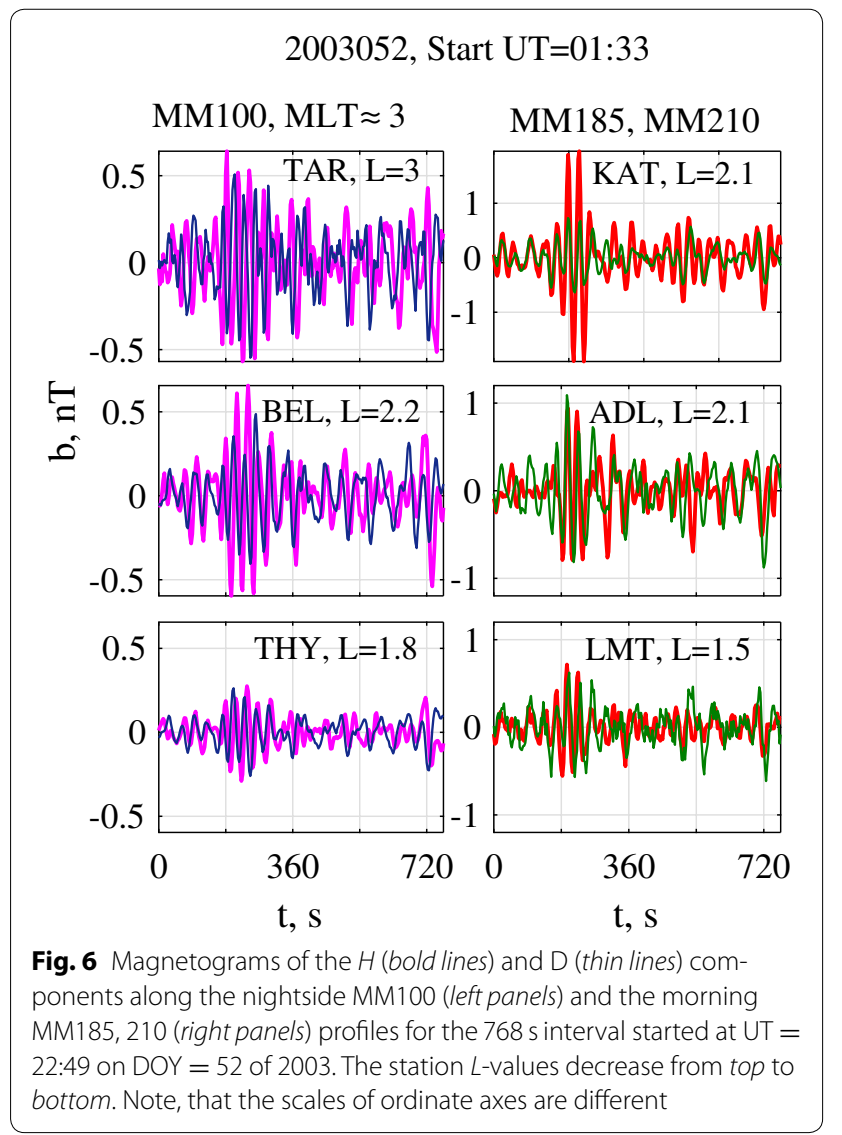

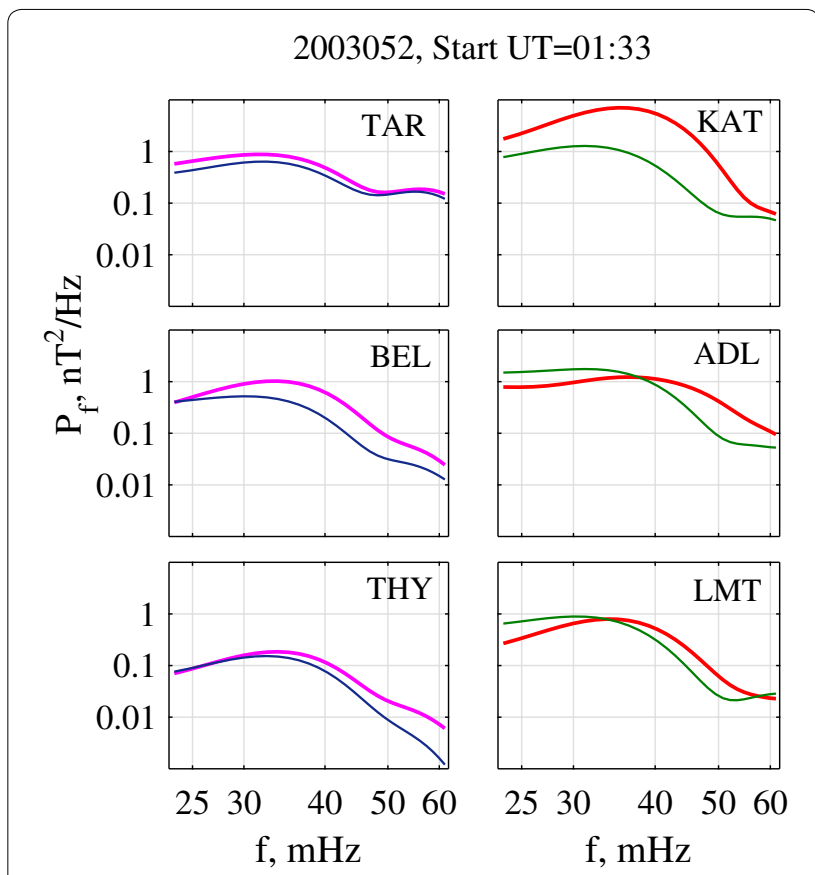

Fig. 7 PSD $P_{f}$ for the event shown in Fig. 6. H/D components are shown with bold/thin lines

same $L$-shell. For this event, the predicted $\left(f_{B}=24 \mathrm{mHz}\right.$ determined from the averaged over 12-min interval $B=4 \mathrm{nT})$ and registered $\left(f_{c}=33 \mathrm{mHz}\right)$ values of central frequency differ at $9 \mathrm{mHz}$, i.e., at about $30 \%$ of the registered frequency.

Coherence spectra for this event are presented in Fig. 8. They indicate a large spatial scale of the Pc3 wave both in the meridional and in the azimuthal directions. At the central frequency $f=33 \mathrm{mHz}$, the maximal values of $\gamma^{2}>0.5$ even for station pairs separated by $5-8 \mathrm{~h}$ in MLT.

\section{Nighttime Pc3 event on DOY $=117$}

Now we consider an example of Pc3 waves recorded on the nightside along the MM100 profile (MLT $\approx 1.5$ ) with no clear correspondence in morning Pc3 waves at the MM185 profile (MLT $\approx 6.5)$ and MM210 (MLT $\approx 09$ ). The pulsation was recorded during time interval started at $2309 \mathrm{UT}$ on DOY $=117$. High-pass-filtered magnetograms of both horizontal components are shown in Fig. 9. The most evident Pc3 signal with a visible period of about $40 \mathrm{~s}$ and peak-to-peak amplitudes about $1 \mathrm{nT}$ was recorded at $L=3$ (TAR) and $L=2.2$ (BEL) stations on the nightside. For this event, D component amplitude is even higher than that of $\mathrm{H}$ component. Similar signal with a lower $(\sim 0.5 \mathrm{nT})$ peak-to-peak amplitude is seen at the lower latitude station THY $(L=1.8)$. Pulsations registered simultaneously in the morning sector have lower amplitudes and look noisier, especially in $\mathrm{H}$ component. 

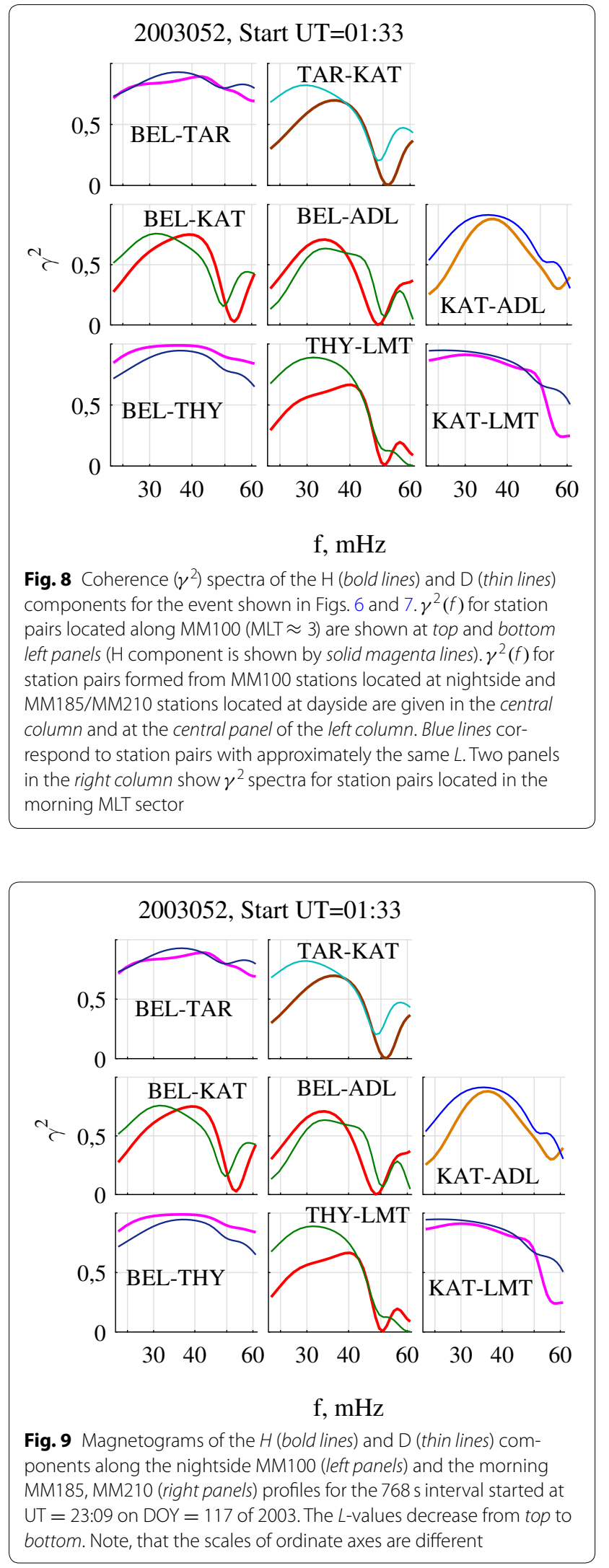

Spectra of the nighttime pulsations have two maxima at $f_{c 1} \approx 22 \mathrm{mHz}$ and $f_{c 2} \approx 45 \mathrm{mHz}$ (Fig. 10). Spectral content of morning Pc3 pulsations is similar, but $\mathrm{H}$ component PSD at $f_{c 1}$ is lower almost by an order of magnitude. The difference in D component PSD is lower, so that night to morning PSD ratio does not exceed 3. For this time interval, the predicted frequency $f_{B}=44 \mathrm{mHz}$, as the average value of IMF magnetic field magnitude is $B=7.3 \mathrm{nT}$. The low-frequency maximum dominating in the night MLT sector lies far away from this value, while the $f_{c 2}$ frequency of the second (minor) maximum agrees with the predicted value.

Coherence spectra for this event are presented in Fig. 11. This event differs from the previous one in the azimuthal coherence scale. Maximal values of $\gamma^{2}$ exceed 0.5 only for the station pairs, formed from stations located at approximately the same magnetic meridian (quasimeridional station pairs) both in the night MLT sector (BEL-TAR and BEL-THY) and in the morning MLT sector (KAT-LMT), and for the ADL-KAT quasi-latitudinal station pair with the stations located in the morning MLT sector and separated at $2 \mathrm{~h}$ in MLT. Stations separated by 5-8 h in MLT demonstrate low coherence. Thus for this event, nighttime and morning pulsations are decoupled.

\section{Statistical relationships}

Diurnal variation of the $\mathrm{Pc} 3$ occurrence rate at different L-shells

The signal's occurrence rates from any automatic detection algorithm depend on the selection procedure,

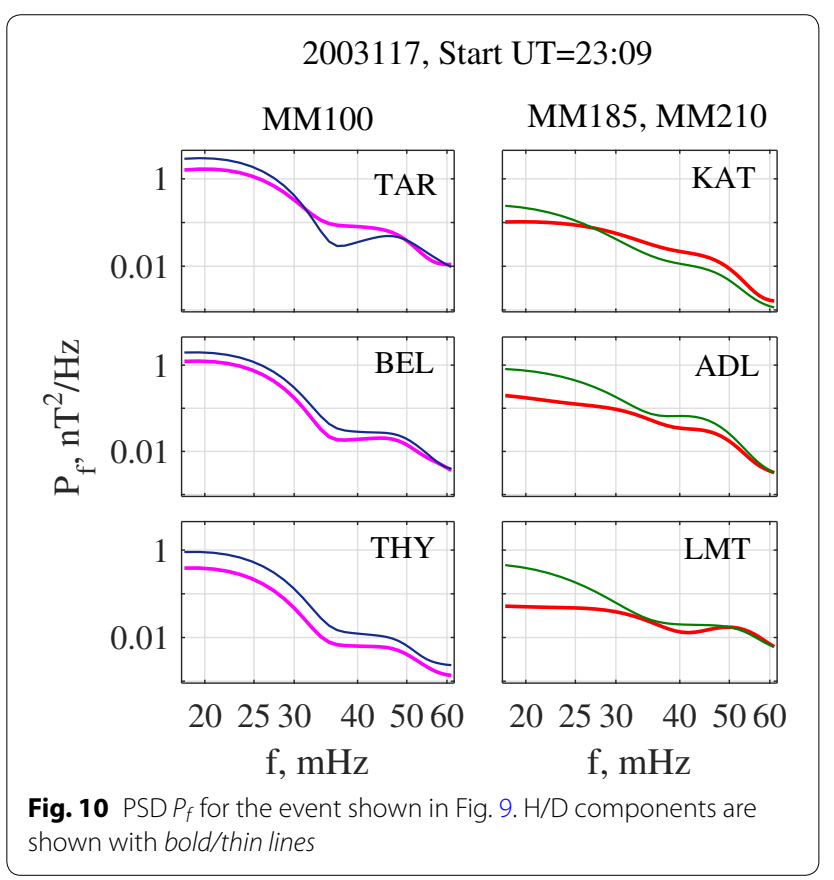




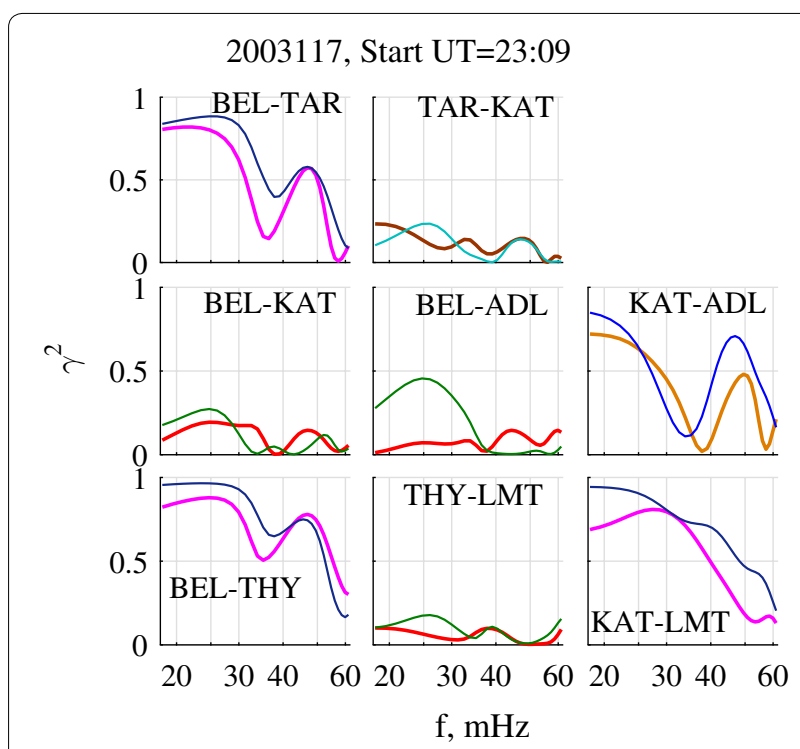

Fig. 11 Coherence $\left(\gamma^{2}\right)$ spectra of the $\mathrm{H}$ (bold lines) and $\mathrm{D}$ (thin lines) components for the event shown in Figs. 9 and 10. The format of the figure is the same as that of Fig. 8

parameters of detection, and the interference level at an observatory. After the set of parameters is fixed, a valid procedure should lead to results that coincide qualitatively with known from other methods, at least for a wellknown object like daytime Pc3 pulsations.

To illustrate the relation between nighttime Pc3s studied here and typical daytime Pc3s, the yearly averaged of the Pc3 occurrence rate diurnal variation calculated in a 4-h window is presented in Fig. 12 from $L=3$ (TAR) to the dip equator (GLP, BCL). Data for the whole year of observations have been used for all stations except the two equatorial sites, for which only four-month data (days $1-120$ ) are available. The occurrence rate $P$ is defined as a ratio of the number of Pc3 intervals to the total number of intervals with valid data in the corresponding MLT sector and night to day occurrence ratio $R$ is calculated as a ratio of $P$ in $0-4$ to that in $8-12$ MLT sector.

Diurnal variation at TAR is typical for the midlatitude Pc3 pulsations with clear pre-noon maximum $P=0.33$ in 8-12 MLT sector both in $\mathrm{H}$ and in D components. In the post-midnight sector ( $0-4 \mathrm{MLT}) P=0.045$, i.e., it is lower by almost an order of magnitude. At $L \approx 2$ (BEL and KAT), the resonant amplification manifests itself in the highest along the profile occurrence rate in $\mathrm{H}$ component and in a wider dayside (from 8 to 16 MLT) maximum in $\mathrm{H}$ component. At BEL the maximal $P>0.5 \mathrm{in} \mathrm{H}$ component and $P=0.33$ in $\mathrm{D}$ component. In the postmidnight sector $P=0.05$ in both components, i.e., the night/day occurrence ratio is nearly the same as at TAR in $\mathrm{D}$ component. It is lower in $\mathrm{H}$ component because of field line resonance effect, which is maximal at dayside in $\mathrm{H}$ component. At the KAT in the Southern hemisphere, $P=0.02$ in $0-4$ MLT sector, and in 8-12 MLT sector it reaches $0.16(0.3)$ for $D(H)$ components. Note that in $\mathrm{D}$ component night/day occurrence ratio $R \approx 0.13$ for all three midlatitude stations, while in $\mathrm{H}$ component it is influenced by local effects. The other important point is that relative variables, like night/day ratio, are more stable than absolute values of the occurrence rate.

Pc3 occurrence rate decreases with the decrease of $L$ both at day and at nighttime. Daily maximal $P$ at THY ( $L$ $=1.8$ ) are 0.2 and 0.14 in $\mathrm{H}$ and $\mathrm{D}$ components, respectively, while in the post-midnight sector they reach 0.013 and 0.02 , i.e., night/day occurrence ratio is only $R$ $=0.06$ in $\mathrm{H}$ component and $R=0.14$ in $\mathrm{D}$ component. The decrease in dayside Pc3 occurrence is seen further at LMT $(L=1.45)$ where the daily maximal $P=0.06(0.07)$ for $\mathrm{H}(\mathrm{D})$ components, while in the post-midnight sector $P=0.02$ for both components and $R=0.33(0.25)$ for $\mathrm{H}$ (D) component.

Near the geomagnetic equator, D component amplitude and Pc3 occurrence vanish, while in $\mathrm{H}$ component the effect of equatorial enhancement leads to an increased Pc3 occurrence rate, especially in the early morning sector. At GLP station daily maximal $P=0.08$ and in the post-midnight sector $P=0.02$. At the $\mathrm{BCL}$ station, located nearly at the same magnetic meridian as KAT and LMT, daily maximal $P=0.11$ and $P=0.025$ in the post-midnight sector, exceeding the corresponding values at the non-equatorial low-latitude station LMT.

Hence, one can see from the analysis of the diurnal variation that yearly averaged night/day Pc3 occurrence ratio is $R$ varied from 0.12 to 0.14 in $\mathrm{D}$ component at middle latitudes and in $\mathrm{H}$ component at $L=3$ (TAR). Near the L-shell where field line resonance for $f \approx 30 \mathrm{mHz}$ Pc3 pulsations is found under typical conditions $(L \approx 2)$, the values of both nighttime and daytime occurrences in the $\mathrm{H}$ component are maximal. However, the night/day occurrence ratio $R$ has a minimum due to the resonant enhancement at the dayside. At the equatorial latitudes, the Pc3 pulsations are almost linearly polarized along the magnetic meridian, and night/day occurrence ratio is higher than that at low latitudes away from the geomagnetic equator.

In this subsection, we have verified the Pc detection algorithm on diurnal variations of Pc3 occurrence rate. This test shows that everywhere from middle to equatorial latitudes well-known regularities for classical daytime Pc3s are reproduced and that the algorithm can be used for a statistical analysis of nighttime Pc3s. 


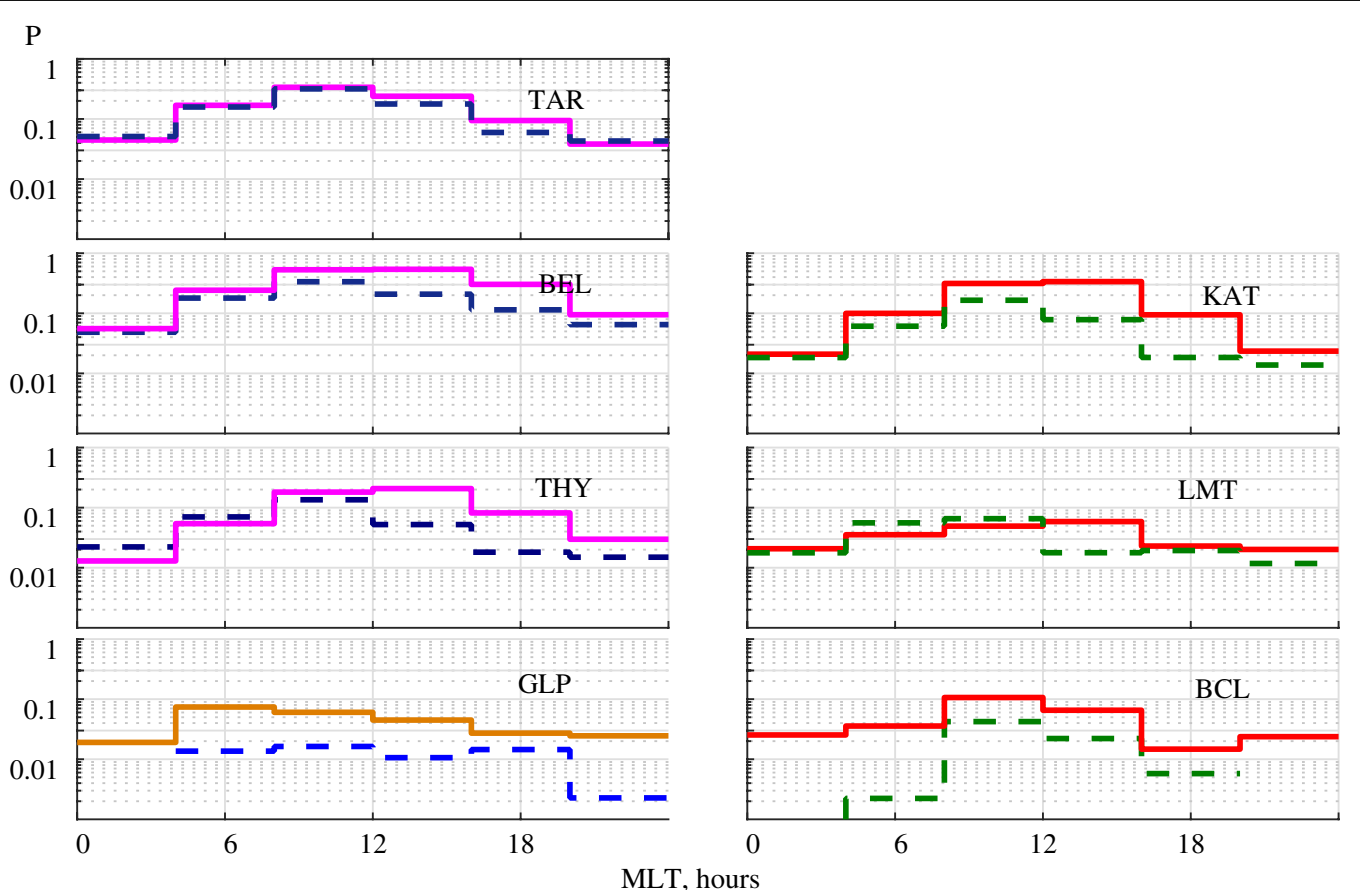

Fig. 12 Yearly averaged diurnal variation of $\mathrm{PC} 3$ occurrence rate $P$ for stations at $L \approx 2$ (top panel), $1.45<L<1.83$ (middle panel), and $L \approx 1$ (bottom panel). H/D components are shown with solid/dashed lines. Colors correspond to different geomagnetic longitudes

\section{Nighttime Pc3 occurrence rate and solar wind velocity}

Nighttime Pc3s are not observed every night, and so a question arises about space weather conditions favorable for their excitation. The occurrence rate $P$ has been calculated as the ratio of the number of Pc3 intervals to the total number of intervals with valid data in the 21-04 MLT sector. The daily averaged $P$ is calculated for a fourmonth interval during the year 2003 (DOY $=1-120$ ) to formulate a hypothesis about factors controlling night Pc3 occurrence. Then it is verified on an extended 12-month interval.

For this analysis, two midlatitude (BEL, KAT) and two equatorial (BCL, GLP) stations are chosen at $L$-values where the amplitude maxima of night Pc3s were found in case studies. These two pairs of stations are separated in MLT by $5-10 \mathrm{~h}$. Variations of $P$ for these two station pairs for DOY $=1-120$ of 2003 are shown in Fig. 13. The background occurrence rate $P$ of the nighttime Pc3s at the equatorial stations is generally negligible, but several clear enhancements on certain days are found (e.g., DOY $=24,51,77,83,101$, and 107). At middle latitudes, $P$ is higher in general. Along with the enhancements seen simultaneously at the equatorial stations, there are several additional peaks seen at both or only at one of the two midlatitude stations.

First, we analyze the peaks found in the occurrence rate simultaneously at several stations. Correlated variations of $P$ of nighttime Pc3s suggest that some global factor controls their generation. At least, for some of nighttime Pc3 a morning counterpart with higher amplitudes exists in the morning MLT sector. An example of coherent Pc3 pulsations recorded simultaneously in the morning and night MLT sectors is shown in Figs. 6, 7 and 8. A growth of typical dayside Pc3 wave amplitude with the solar wind velocity $V$ is a well-established fact (e.g., Guglielmi and Troitskaya 1974; Singer et al. 1977; Greenstadt et al. 1979). Thus, occurrence of global nighttime Pc3 pulsations may be controlled by solar wind speed.

Variations of the solar wind speed $V$ are shown at the bottom panel of Fig. 13. Global peaks of $P$ are recorded on days with high solar wind speed $(V>500 \mathrm{~km} / \mathrm{s})$, and for several intervals it reaches $700 \mathrm{~km} / \mathrm{s}$. This correspondence naturally leads to a suggestion that night Pc3 occurrence is controlled by the solar wind speed.

To check this hypothesis, we selected automatically highly coherent Pc3 events recorded at BEL and KAT (same L-shell) in 2003, for which the BEL station was on the nightside, while KAT was on the day side, and when the day side amplitude was larger than the nightside one. The selected time interval $22-2 \mathrm{UT}$ corresponds to MLT intervals 0-4 for BEL and 5.5-9.5 for KAT. Whenever spectral coherence between BEL-KAT exceeds its boundary values $\gamma^{2}>\gamma_{B}^{2}$ and the power spectral density at nightside is lower than that in the at the dayside, 


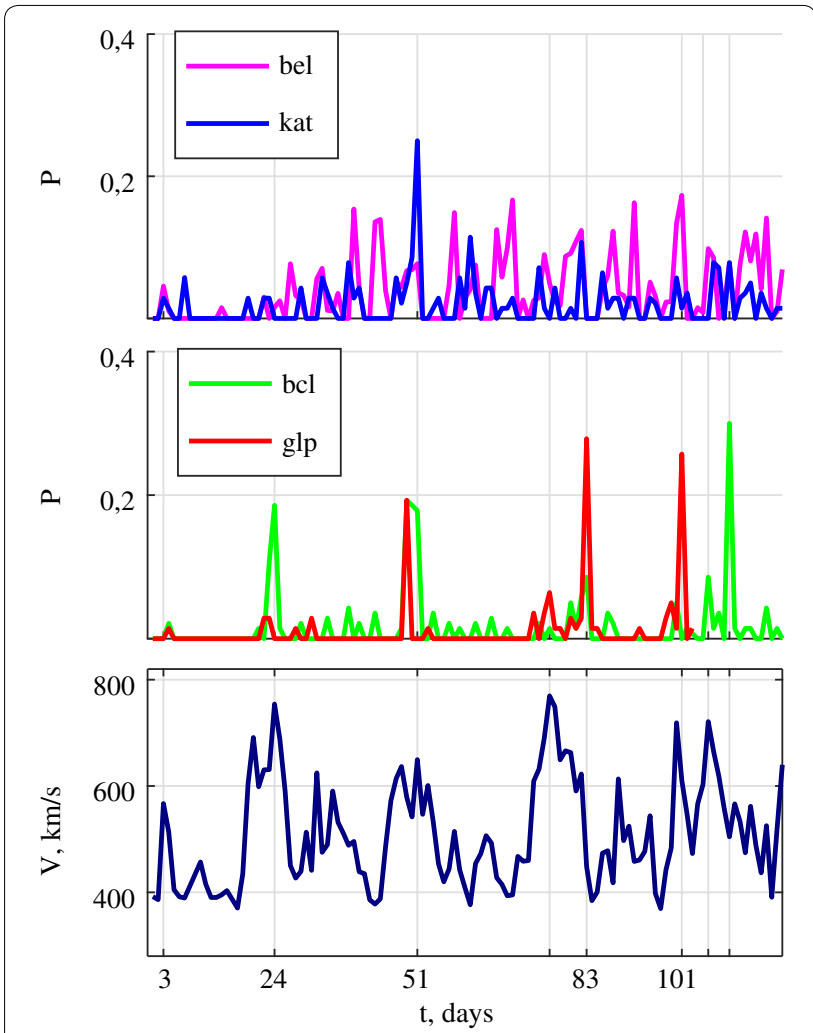

Fig. 13 Occurrence rate $P$ of nighttime Pc3 pulsations for January to April of 2003 for two midlatitude (BEL and KAT) (upper panel), and two equatorial ( $B C L$ and GLP) (middle panel) stations. Bottom panel shows the variations of solar wind velocity

$P_{f}^{n}<P_{f}^{d}$, the Pc3 is classified as nighttime Pc3 pulsations of dayside origin $\left(\mathrm{Pc} 3 \mathrm{n}_{D}\right)$. If, on the contrary, $\gamma^{2}<\gamma_{B}^{2}$ or $P_{f}^{n}>P_{f}^{d}$, pulsations have been classified as local night Pc3s $\left(\operatorname{Pc}_{3} \mathrm{n}_{L}\right)$. For this test, a boundary value of coherence $\gamma_{B}^{2}=0.33$ is taken.

The empirical probability density functions $P^{*}$ for the intervals with night $\mathrm{Pc} 3 \mathrm{~s}$ of dayside origin $\mathrm{Pc}_{D} \mathrm{n}_{D}$, local night Pc3s Pc3n $\mathrm{n}_{L}$, and all the $22-2 \mathrm{UT}$ intervals during the year against solar wind speed are shown in Fig. 14. The

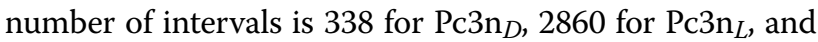
more than 10,000 for the general sample. It is seen from the figure that solar wind speed is higher for the intervals with nighttime Pc3 pulsations of dayside origin than for the general sample. The mean $V$ for $\operatorname{Pc} 3 n_{D}$ intervals is $615 \mathrm{~km} / \mathrm{s}$, and it is equal to 554 and $550 \mathrm{~km} / \mathrm{s}$ for $\operatorname{Pc} 3 \mathrm{n}_{L}$ and for the general sample, respectively. However, not all the $V$ values in the samples are independent. To exclude highly correlated values of $V$ corresponding to series of Pc3 events, only the intervals separated by $12 \mathrm{~h}$ or more from the end of the previous interval were taken as independent events. After that the number of intervals is reduced to 105 for the $\mathrm{Pc} 3 \mathrm{n}_{D}$ sample. The mean solar wind speed for this subsample is equal to $595 \mathrm{~km} / \mathrm{s}$, and thus, the difference

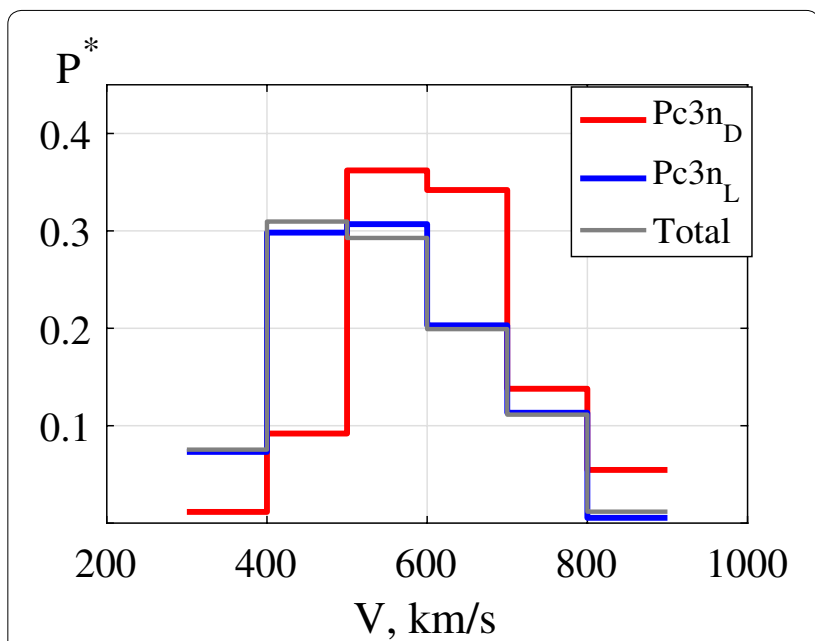

Fig. 14 Empirical probability density function of the solar wind speed for the intervals with the two types of night Pc3 pulsations: $P C 3 n_{D}$ (red) and $P C 3 n_{L}$ (blue), and for all the intervals analyzed (gray)

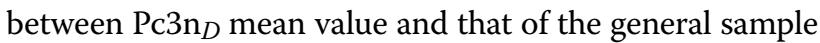
is meaningful at better than $1 \%$ confidence level.

\section{IMF control}

The other test for the dayside origin of nighttime Pc3s of the first group is the analysis of correspondence of registered central frequency $f_{C}$ to the frequency $f_{B}$ predicted from (1). $P^{*}$ distribution over IMF magnetic field magnitude $B$ for the same three groups of intervals as in Fig. 14 is shown at the left panel of Fig. 15. It is seen from the histogram, that $6<B<9 \mathrm{nT}$ values, corresponding to Pc3 frequency range $(36-54 \mathrm{mHz})$, is found to be more often during $\mathrm{Pc}_{\mathrm{C}} \mathrm{n}_{D}$ intervals than during the $\mathrm{Pc} 3 \mathrm{n}_{L}$ intervals and for the general sample. For a more accurate comparison of registered and predicted Pc3 frequencies for the two groups of night Pc3s, $P^{*}$ distribution over the frequency mismatch $\Delta f=f_{B}-f_{c}$ is calculated and shown at the right panel of Fig. 15. The most important result is that both distributions are centered at zero $\Delta f$. Possible reasons for this effect will be discussed in "Discussion" section. The main difference in these two distributions is

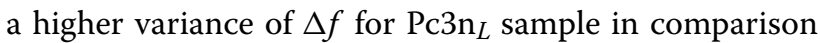

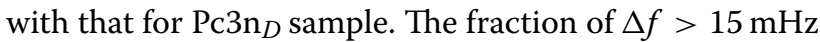
is about two times higher for ${\operatorname{Pc} 3 n_{L}}$ than for $\operatorname{Pc} 3 n_{D}$. The

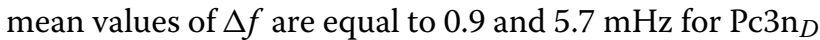
and $\operatorname{Pc} 3 \mathrm{n}_{L}$, respectively. This result is meaningful at better than $1 \%$ confidence level.

With these two tests, we have found that night Pc3s of day origin, selected only with amplitude and coherence criterion, demonstrate features typical for dayside Pc3s and nighttime Pc3 pulsations of intermediate and high frequencies studied in Villante and Tiberi (2016) at $L=1.6$. On the contrary, the $V$ and $B$ distributions 

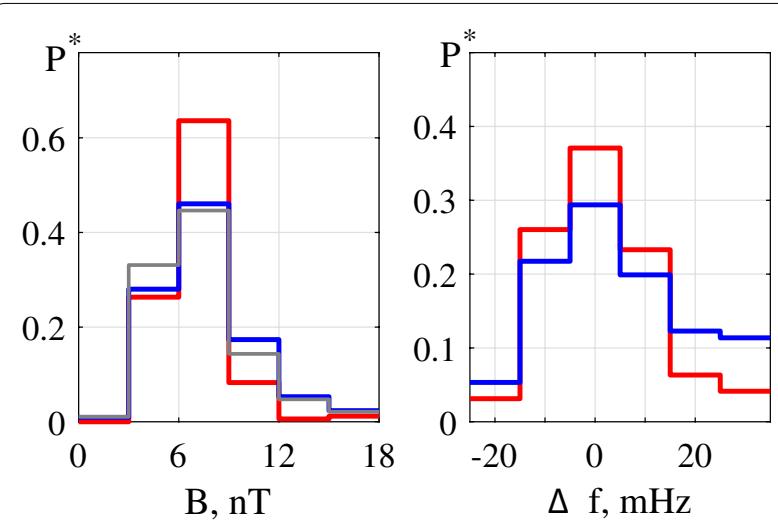

Fig. 15 Left empirical probability density function of the IMF magnetic field magnitude $B$ for the intervals with the two types of night PC3 pulsations: $\mathrm{Pc}_{\mathrm{C}} \mathrm{n}_{D}$ (red) and $\mathrm{Pc} 3 \mathrm{n}_{L}$ (b/ue), and for all the intervals analyzed (gray). Right empirical probability density function of $\Delta f=f_{B}-f_{C}$ for the intervals with the two types of night Pc3 pulsations: $\operatorname{Pc} 3 n_{D}$ (red) and $\operatorname{Pc} 3 n_{L}$ (blue)

for the intervals of local night Pc3s agree with the mean annual distributions of these parameters. This means that the occurrence of this second type of nightside Pc3 pulsations should be controlled by some other factor.

\section{Local nightside Pc3 pulsations and auroral activity}

An auroral substorm is the most intense nighttime disturbance and should be examined as a likely candidate for energy source of local night Pc3 pulsations at middle latitudes. The variations of AE index in January to April of 2003 are shown in Fig. 16. Days, when local night Pc3 pulsations were recorded at BEL, are shown with stars. Four of five most powerful auroral activations are marked also with the occurrence of local night Pc3 pulsations (days 36-40, 57-66, 88-95, and 110-117).

To check the relation between local night Pc3 pulsations and auroral activity, we analyze the distribution of $\mathrm{AE}$ index for the same samples as we did for $V$ and IMF. Figure 17 shows $P^{*}$ distribution over AE. The difference

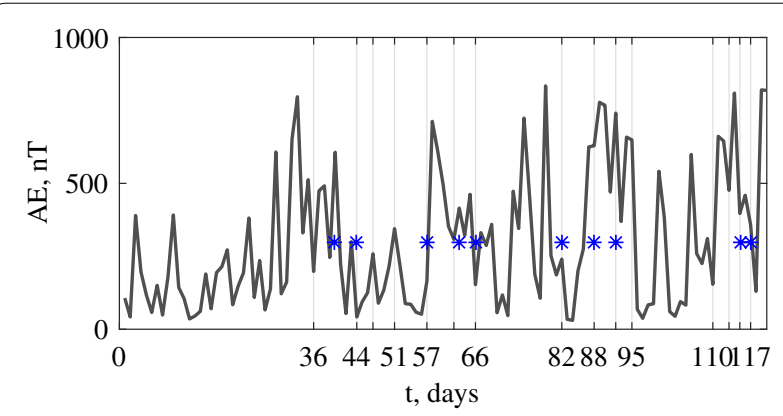

Fig. 16 Variation of daily averaged AE index in January to April of 2003. Days when local night PC3 pulsations were recorded at BEL are indicated with stars between the distributions for the general sample, on the one hand, and for $\operatorname{Pc} 3 \mathrm{n}_{D}$ and $\operatorname{Pc}_{C} 3 \mathrm{n}_{L}$ samples, on the other hand, is maximal at low values of auroral activity $\mathrm{AE}$ $<250 \mathrm{nT}$. Night Pc3s are registered only rarely at low AE, although these $\mathrm{AE}$ values dominate in the general sample. The difference between two types of night Pc3s becomes essential at high auroral activities $(\mathrm{AE}>1000 \mathrm{nT})$. The probability of $(\mathrm{AE}>1000 \mathrm{nT})$ for $\mathrm{Pc} 3 \mathrm{n}_{L}$ sample is almost three times higher than for $\operatorname{Pc} 3 \mathrm{n}_{D}$ one.

However, nighttime Pc3s occur at any level of auroral activity, beginning with very low $\mathrm{AE}$, i.e., there is no threshold value of AE for their generation. Local activations, which do not contribute to general auroral activity characterized by $\mathrm{AE}$, may provide energy for nighttime Pc3s. To discriminate between local and global effects in night Pc3s, we study a correlation between variations of night Pc3 occurrence rates and local geomagnetic activity at two station pairs: BEL-TAR in Europe and KAT-ADL in Australia. These two station pairs are located approximately along the same geomagnetic longitude and latitude, respectively. The first four months of the year 2003, when the data coverage is the best, are used for the analysis. Local activity coefficient $K$ is introduced as the logarithm of PSD in the $0.3-0.5 \mathrm{mHz}$ frequency range (time scales from 33 to $55 \mathrm{~min}$ ). The coefficient $K$ characterizes the intensity of local bay-like geomagnetic disturbances at an observation cite. Linear correlation coefficients $C_{P-K}$ between variations of $K$ and Pc3 occurrence $P$ are given in Table 2. For both BEL and KAT stations, $C_{P-K}$ is maximal for $K$ calculated at the same point as $P$. It decreases with distance if $K$ is taken at a point shifted in the azimuthal (BEL-KAT, BEL-ADL) and meridional (BEL-TAR) directions. This means that generation of nighttime Pc3s is controlled not only by the auroral activations but also by more local activations, which are recorded only at short distances from the observational point.

\section{Seasonal effects and terminator position}

The results of the previous analysis have been given in $L$ MLT coordinates. The examples shown in Figs. 3, 4, 5, 9, 10 and 11 show the Pc3 pulsations in the post-midnight MLT sector at locations separated by more than $3 \mathrm{~h}$ from the terminators, while the event shown in Figs. 6, 7 and 8 belongs to pre-morning sector. In the yearly averaged statistical results, the same MLT corresponds to different positions of an observation site and its conjugated point with respect to the terminator. To estimate influence of the ionosphere conditions on night Pc3 parameters, we have calculated Pc3 occurrence rate and the parameters used for the discrimination between the two types of night Pc3s, i.e., daynight spectral coherence and PSD ratio. The calculations have been done separately for winter and summer seasons 


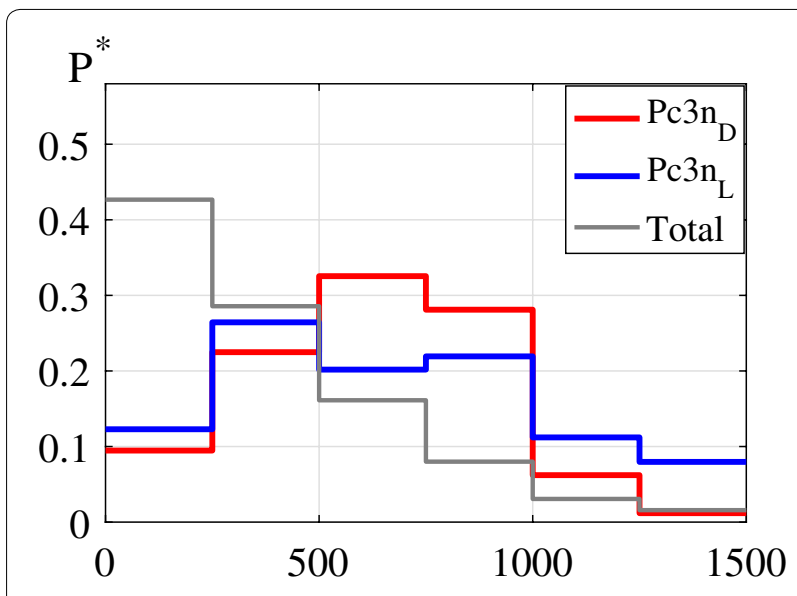

AE, nT

Fig. 17 Empirical probability density function of $A E$ for the intervals with the two types of night Pc3 pulsations: $P_{C} 3 n_{D}$ (red) and Pc $3 n_{L}$ (blue), and for all the intervals analyzed (gray)

Table 2 Correlation coefficients between night Pc3 occurrence rate and local geomagnetic activity

\begin{tabular}{lllll}
\hline Station & BEL & TAR & KAT & ADL \\
\hline BEL & 0.45 & 0.40 & 0.34 & 0.34 \\
KAT & 0.24 & 0.26 & 0.31 & 0.24 \\
\hline
\end{tabular}

and for different positions of the observational point with respect to the dawn terminator. We used data from BEL station, where the latitudinal maximum of Pc3 occurrence has been found, and the KAT station, located in the Southern hemisphere nearly at the same L-shell and separated by $5.5 \mathrm{~h}$, from BEL. The latter station has been taken to analyze the night-day spectral coherence and PSD ratio.

The results of the analysis are shown in Fig. 18. The zero of time axis $\tau=0$ is taken as a later moment from two time instants: the dawn terminator at the observational point (BEL), or at the conjugated point. Thus, $\tau=0$ indicates the moment when both footprints of the field line move to dayside. All the Pc3s detected in $[-6,2]$ $\mathrm{h}$ interval have been divided into two groups in accordance with BEL position in the winter (index $w$ in Fig. 18) or summer (index $s$ in Fig. 18) hemispheres. Then each of the groups is again divided into two subgroups in accordance with the solar illumination at the observational and the conjugated points. For the first group of events, both points are at the dark side $(\mathrm{nn}=$ night-night index in Fig. 18), and the second group includes events occurred when one of the footprints is at the dayside (nd = nightday index in Fig. 18). The upper panel shows the averaged Pc3 occurrence rate variation. Averaged $\gamma^{2}$ for BEL-KAT station pair and BEL/KAT (night/day) PSD ratio $R$ for

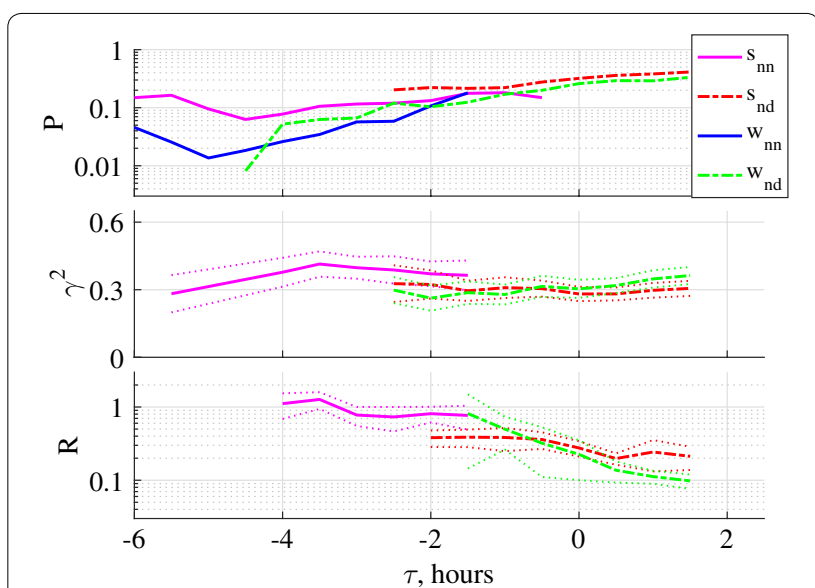

Fig. 18 Averaged variations of Pc3 parameters from night to morning hours at BEL and KAT stations $(L \approx 2)$. PC3 occurrence rate $P$ is given at the upper panel, day-night spectral coherence is given at the middle pair, and BEL/KAT (night to day) PSD ratio are given at the bottom panel. Solid/dashed lines show the results for the night position of both footprints (nn)/ dayside position of one of footprints (nd). Northern summer/winter are shown with warm/cold colors

$\gamma^{2} \geq 0.33$ events are given at the middle and bottom panels, respectively.

The upper panel of Fig. 18 shows that Pc3 pulsations occur not only near the terminator, but deep at night, as well. Seasonal effect becomes more evident from dawn to midnight and for the intervals when both footprints are at nightside. Summer to winter occurrence ratio for these "night-night" Pc3 pulsations varies from about 1 at $\tau=-2 \mathrm{~h}$ to almost 10 at $\tau<-5 \mathrm{~h}$. When one of the footprints is at the dayside, the summer/winter occurrence ratio does not exceed 2 .

$\gamma^{2}$ and $R$ variations are shown in Fig. 18 for the time intervals, when $N>30$ events were recorded with the $95 \%$ confidence intervals shown with dotted lines. The "night-night" Pc3s are rarely registered in the winter hemisphere, and thus, there are not enough events for the statistical analysis. These parameters under "night-night" conditions are estimated only in the summer hemisphere. If one of the footprints is at dayside, the number of events exceeds the threshold value in both hemispheres. The ionospheric conditions for the nigh-day pulsations correspond to quarter-wave resonance generation along the field line (Allan 1983). The coherence between day and day-night Pc3s almost coincides in two hemispheres, and it is lower than the coherence between day and pure night pulsations. This result naturally follows from different symmetry and expected frequencies for half-wave and quarter-wave pulsations.

Night to day PSD ratio $R$ has been calculated for coherent Pc3 pulsations $\left(\gamma^{2} \geq 0.33\right)$ and is shown in 
the bottom panel of Fig. 18. Pc3 pulsations of dawn origin correspond to $R<1$. These pulsations are typical at $\tau>-3.5 \mathrm{~h}$ under both night-night and day-night (quarter-wave) conditions. Local night Pc3 dominates at earlier MLT.

\section{Phase difference dependence on MLT for nighttime Pc3 and $\mathrm{Pi} 2$ pulsations}

Nighttime Pc3s differ from Pi2s not only in periods, but also in spatial distribution of wave amplitude, coherence, and phase. To illustrate this difference, we analyze the phase difference between pulsations at the ADL and KAT stations located at $L=2.1$ and separated by $2 \mathrm{~h}$ in MLT. Pi2 pulsations were selected with a similar detection technique, as used for Pc3s, and the influence of nighttime Pc4 pulsations is minimized by the choice of an amplitude threshold, high enough to exclude the influence of nighttime Pc4s which are essentially less intensive.

The results for the four-month interval (days 1-120) are shown in Fig. 19. For time intervals with $\gamma^{2}>0.6$, empirical probability density functions $P^{*}$ are given by colors in dependence on MLT and phase difference $\Delta \varphi$. Negative/positive $\Delta \varphi$ values correspond to eastern/western station leading.

The results for both horizontal components in Pi2 and Pc3 pulsations are presented at left and right panels, respectively. Nighttime Pc3 pulsations are characterized by negative phase difference in $\mathrm{H}$ component (upper right panel), while $\mathrm{D}$ component pulsations are almost in phase (lower right panel). They differ both from typical morning Pc3s (see MLT> 5, where the phase difference is negative in both components) and from Pi2s which are in phase for both components.

The above analysis of phase difference indicates the existence of a difference in propagation of different types of pulsations, but it cannot be used to conclude about the propagation direction. For that, a more precise analysis of group velocity is required.

\section{Discussion}

Waveforms very similar to usual dayside Pc3 have been found at nightside with occurrence rates depending on the geomagnetic latitude, magnetic and solar local times,

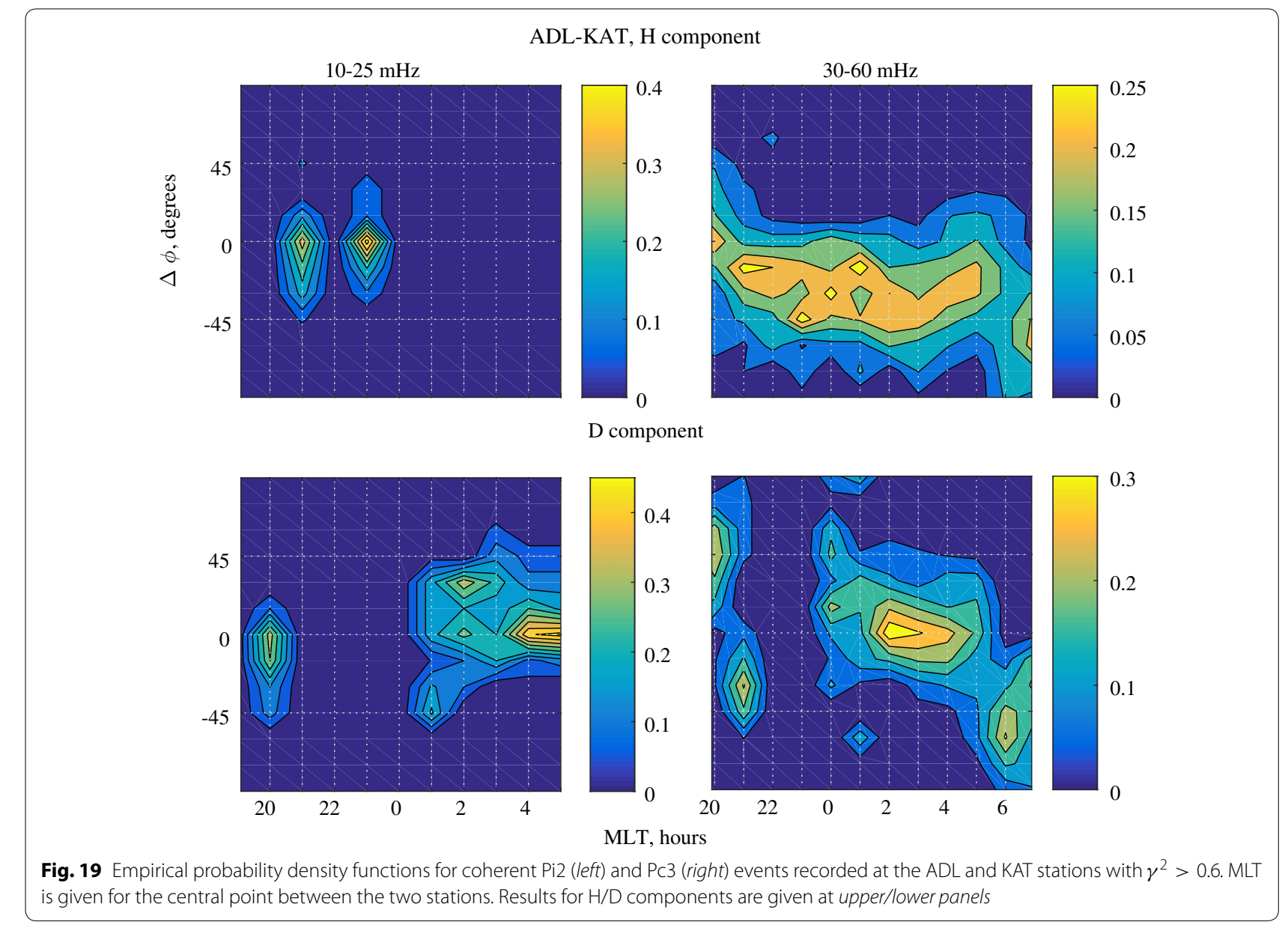


and space weather conditions. Nighttime Pc3s have been divided into two groups according to their amplitude and coherence distribution along the geomagnetic latitude. The space weather conditions were found to be different for these two groups, and thus, one can assume different generation mechanisms for them.

The first mechanism is the refraction of fast compressional waves propagating from the upstream region through the dayside magnetosphere to the nightside. In this case, the amplitudes of the nightside Pc3s are to be lower than the amplitudes of their dayside counterparts. The channel of the Pc3 wave energy transmission from the dayside to the nightside might be a waveguide near the plasmapause, formed owing to the minimum of Alfven velocity in the region inside the plasmasphere dome Gul'elmi (1970). However, a quantitative prediction of the rate of the ULF wave energy leakage into the nightside magnetosphere encounters a lack of an adequate 3D MHD wave model.

Equatorial enhancement, found here in nighttime Pc3s, is well known in usual Pc3 (Sarma and Sastry 1995; Matsuoka et al. 1997; Roy and Rao 1998) and Pi2 (Shinohara et al. 1998) pulsations. A possible mechanism that is responsible for the equatorial peak wave amplitude can involve the enhancement of the ionospheric currents induced by Alfven waves as they spread into the equatorial ionosphere with the elevated Cowling conductance (Yumoto et al. 1992). Another possible mechanism is the direct penetration of compressional wave energy toward the near-equatorial region (Yumoto et al. 1985; Waters et al. 2000; Heilig et al. 2007; Sutcliffe et al. 2013). Active generation of magnetohydrodynamic (MHD) waves along the ionosphere by fluctuations of the equatorial electrojet can also contribute to the near-equatorial ULF wave activity (Fedorov et al. 1999).

Our study confirms the conclusion of Villante and Tiberi (2016) on the key role of foreshock waves in formation of night Pc3 energy spectrum. A good correspondence between predicted and observed Pc3 wave central frequencies is found for $\operatorname{Pc} 3 \mathrm{n}_{D}$ population, and to some extent for $\mathrm{Pc} 3 \mathrm{n}_{L}$ groups as well. Partly, it may be due to a similar mechanism of wave energy leakage from the dusk side. Although our analysis of evening-night spectral coherence and PSD ratio has not revealed pulsations with the same properties as at the morning side, we cannot claim that such a mechanism is impossible if a shorter base in the azimuthal direction is taken. Also foreshock waves in Pc3 frequency range can play a role of a band-limited source even in the absence of conditions for their effective penetration into the magnetosphere. A possibility of such a scenario is indirectly confirmed by an essential difference between registered and predicted central frequencies for the event on day 2003052 and by statistical results of Villante et al. (1999). In Villante et al. (1999), it was found that observed Pc3 central frequency was correlated with $B$ under highly variable IMF, but the difference between $f_{c}$ and $f_{B}$ during the Pc3 event on day 2003052 lies within the spread of frequencies shown in Fig. 4 of Villante et al. (1999). Notice that the first hours of the day 2003052 were also characterized by highly variable IMF.

Local nighttime Pc3s can be generated under moderate solar wind speed, and they are probably generated by a source inside the magnetosphere. Our analysis of auroral activity for the days when nighttime Pc3s are registered at middle latitudes has shown that a fraction of intervals with high $(\mathrm{AE}>1000 \mathrm{nT})$ auroral activity for local night Pc3 pulsations $\left(\operatorname{Pc}_{2} n_{L}\right)$ exceeds that for night Pc3 of day origin $\left(\operatorname{Pc} 3 \mathrm{n}_{D}\right)$ and for the general sample. However, night Pc3 pulsations occur at any AE level beginning with very low $A E$ values. We have found that local geomagnetic disturbances that do not contribute to $\mathrm{AE}$ index may also be a source of night Pc3 pulsations. This conclusion is confirmed by the local nature of correlation between geomagnetic activity with timescales of 30-50 min and the $\mathrm{Pc} 3 \mathrm{n}_{L}$ occurrence rate.

A similar driving of non-substorm Pi2 waves and poleward auroral boundary intensifications by irregular magnetotail activity was found in Sutcliffe and Lyons (2002), Kwon et al. (2013). The wave energy transfer from the magnetotail to the ground is probably performed via the fast mode. Period of nighttime Pc3 waves is to be determined either by the periodicity of energy release process, or by band-pass filtering which can occur during the wave trapping and propagation along the magnetotail waveguide (Mazur et al. 2010). Finally, there is a theoretical prediction (Mazur and Leonovich 2006) that a largescale minimum of Alfven velocity on the nightside of the magnetosphere can be a cavity for MHD modes resulting in a quasi-periodic response to the nightside magnetospheric activity. However, parameters and eigenfrequencies of this cavity are known very approximately, and it is unclear whether they may comprise the Pc3 band. Coordinated observations at low-orbiting CHAMP satellite and on the ground (Cuturrufo et al. 2015) indeed confirmed that nighttime Pc3 pulsations at low latitudes are fast mode waves directly transmitted to the ground. Upon propagation into the inner magnetosphere, in contrast to Pi2 transients, fast mode waves in the Pc3 band emitted from the magnetotail region can be partially converted into Alfven waves at midlatitudes $(L \approx 2)$.

An occurrence of quasi-monochromatic waves at the nightside may be influenced by the field line resonances. Appearance of high-quality signals at night seemingly contradicts the popular notion about a severe Alfven wave damping in the magnetospheric resonator 
terminated by low-conductive ionospheres (Hameiri and Kivelson 1991). The occurrence of nightside Pc3 waves was considered in Bulusu et al. (2015) as an indirect argument in favor of the model of an ideally insulating ionosphere, $\Sigma_{P} \ll \Sigma_{A}$. However, the analysis of Pc3 dissipation in Yagova et al. (1999) showed that at middle and low latitudes an approach based on the consideration of height-integrated conductance $\Sigma_{P}$ and nominal Alfven wave conductance $\Sigma_{A}$ only may be misleading. In a realistic magnetosphere with inhomogeneous field-aligned plasma distribution, an essential part of Alfven wave energy is reflected above the E-layer (Yagova et al. 1999). As a result, the Pc3 wave absorption in the nightside ionosphere turns out to be much lower than predicted by the "thin ionosphere" model.

Specific nighttime Pc3 waves may be an additional channel of coupling between nighttime magnetospheric activity and low-latitude ionosphere, which requires further studies with the use of magnetotail satellites. There are at least two important problems of nighttime Pc3 generation which are out of our present research but should be solved in the future. The first problem concerns the night Pc3 during geomagnetic storms. Geomagnetic storms reveal themselves in a complicated combination of different types of pulsations, recorded simultaneously (Balasis et al. 2015). Storm-time pulsations demonstrate not only quantitative, but also qualitative differences from non-storm ones (Posch et al. 2003; Manninen et al. 2008). Thus, an accurate interpretation of storm nighttime Pc3s is possible only with the analysis of simultaneous ground and satellite observations, as it was done for dayside pulsations by Balasis et al. (2012, 2015).

The second problem is the relation of this type of pulsations to known current systems and their variations at different timescales. The bibliography on a possible relation of wave geomagnetic phenomena with global current systems, such as auroral and equatorial electrojets, storm-time current systems, and solar quiet system, includes several hundred publications (see e.g., Stening 1995; Hamid et al. 1967). However, this problem is far from being solved. On the one hand, a correlation exists between the intensities of current fluctuations at different frequencies. This result is reproduced both in the well-known correlations of pulsations amplitudes with geomagnetic indexes (Rostoker 1995) and in the analysis with more complicated techniques like empirical mode decomposition (EMD) undertaken by Alberti et al. (2016) for both disturbed and quiet conditions. On the other hand, Pc4 (Kokubun et al. 1989) and lowlatitude Pi2 pulsations (Kwon et al. 2013) are regularly generated at a quiet background. Besides, different current systems are interrelated (Stening 1995); they demonstrate a complicated spatiotemporal evolution (Kamide and Kokubun 1996), resulting in a non-trivial frequency dependence of the corresponding indexes (Takalo et al. 1994; Consolini et al. 1996). Thus, the correlations of $\operatorname{Pc} 3 \mathrm{n}_{L}$ occurrence with $\mathrm{AE}$ and local activity index $K$, found in the present study, can be used only as a qualitative indicator of the existence of an intra-magnetospheric source for these pulsations, but they do not localize this source in a particular current system and/ or specify the parameters of transition processes, necessary for $\mathrm{Pc} 3 \mathrm{n}_{L}$ generation.

\section{Conclusion}

Pc3 waves recorded during nighttime at low/middle latitudes can be classified into two groups. Pc3 waves of the first group are the extension of morning Pc3 pulsations to the nightside. These night signatures of morning Pc3 waves are observed predominantly during the periods of fast solar wind. The second group is composed from locally generated night Pc3 pulsations, which are associated with auroral activations or local geomagnetic disturbances.

\section{Author details \\ ${ }^{1}$ Institute of Physics of the Earth, Moscow, Russia. ${ }^{2}$ Tihany Geophysical Observatory MFGI, Tihany, Hungary. ${ }^{3}$ Space Research Institute, Moscow, Rus- sia. ${ }^{4}$ International Center for Space Weather Science and Education, Kyushu University, Fukuoka, Japan. ${ }^{5}$ Institute of Geophysics of Polish Academy of Sci- ences, Belsk, Duzy, Poland.}

\section{Acknowledgements}

We thank CDAWEB (http://cdaweb.gsfc.nasa.gov/istp_public) for ACE data on solar wind and IMF. AE index is provided by Data Center for Geomagnetism in Kyoto (http://wdc.kugi.kyoto-u.ac.jp/index.html). This work was supported by RFBR Grant 15-55-45064 (NY, VP, NN) and by MEXT/JSPS KAKENHI Grant $15 \mathrm{H} 05815$ (AY, KY).

\section{Publisher's Note}

Springer Nature remains neutral with regard to jurisdictional claims in publishedmaps and institutional affiliations.

Received: 25 January 2017 Accepted: 20 April 2017

Published online: 02 May 2017

\footnotetext{
References

Alberti T, Piersanti M, Vecchio A, De Michelis P, Lepreti F, Carbone V, Primavera L (2016) Identification of the different magnetic field contributions during a geomagnetic storm in magnetospheric and ground observations. Ann Geophys 34:1069-1084. doi:10.5194/angeo-34-1069-2016

Allan W (1983) Quarter-wave ULF pulsations. Planet Space Sci 31:323-330

Balasis G, Daglis IA, Mann IR, Papadimitriou C, Zesta E, Georgiou M, Haagmans R, Tsinganos K (2015) Multi-satellite study of the excitation of Pc3 and PC4-5 ULF waves and their penetration across the plasmapause during the 2003 Halloween superstorm. Ann Geophys 33:1237-1252. doi:10.5194/angeo-33-1237-2015

Balasis G, Daglis IA, Zesta E, Papadimitriou C, Georgiou M, Haagmans R, Tsinganos K (2012) ULF wave activity during the 2003 Halloween superstorm: multipoint observations from CHAMP, Cluster and Geotail missions. Ann Geophys 30:1751-1768. doi:10.5194/angeo-30-1751-2012
} 
Bier EA, Owusu N, Engebretson MJ, Posch JL, Lessard MR, Pilipenko VA (2014) Investigating the IMF cone angle control of Pc3-4 pulsations observed on the ground. J Geophys Res 119. doi:10.1002/2013JA019637

Bulusu J, Sinha AK, Vichare G (2015) An analytic model of toroidal half-wave oscillations: implication on plasma density estimates. J Geophys Res 120:4164-4180

Consolini G, Marcucci MF, Candidi M (1996) Multifractal structure of auroral electrojet index data. Phys Rev Lett 76:482-485

Cuturrufo F, Pilipenko V, Heilig B, Stepanova M, Luhr H, Vega P, Yoshikawa Y (2015) Near-equatorial Pi2 and PC3 waves observed by CHAMP and on SAMBA/MAGDAS stations. Adv Space Res 55:1180-1189

Fedorov E, Pilipenko V, Surkov V, Rao DRK, Yumoto K (1999) Ionospheric propagation of magnetohydrodynamic disturbances from the equatorial electrojet. J Geophys Res 104:4329-4336

Greenstadt EW, Olson JV (1976) Pc3,4 activity and interplanetary field orientation. J Geophys Res 81:5911-5920

Greenstadt EW, Singer HJ, Russell CT, Olson JV (1979) IMF orientation, solar wind velocity, and Pc 3-4 signals: A joint distribution. J Geophys Res 84:527532

Guglielmi AV, Troitskaya VA (1974) Diagnostics of the magnetosphere and interplanetary medium by means of pulsations. Space Sci Rev 16:331-345

Gul'elmi AV (1970) Ring trap for low-frequency waves in the Earth's magnetosphere. Pisma Zh Eksp Teor Fiz 12:35

Hameiri E, Kivelson MG (1991) Magnetospheric waves and the atmosphereionosphere layer. J Geophys Res 96:21125-21134

Hamid NSA, Liu H, Uozumi T, Yumoto K, Veenadhari B, Yoshikawa A, Sanchez JA (1967) Relationship between the equatorial electrojet and global Sq currents at the dip equator region. J Geophys Res 72:2032-2039. doi:10.1029/JZ072i007p02032

Heilig B, Lotz S, Vero J, Sutcliffe P, Reda J, Pajunpaa K, Raita T (2010) Empirically modeled Pc3 activity based on solar wind parameters. Ann Geophys 28:1703-1722

Heilig B, Luhr H, Rother M (2007) Comprehensive study of ULF upstream waves observed in the topside ionosphere by CHAMP and on the ground. Ann Geophys 25:737-754

Jenkins GM, Watts DG (1968) Spectral analysis and its applications. HoldenDay, San-Francisco, Cambridge, London, Amsterdam

Kamide Y, Kokubun S (1996) Two-component auroral electrojet: Importance for substorm studies. J Geophys Res 101:13027-13046

Kay SM (1988) Modern spectral estimation: theory and application. PrenticeHall, San-Francisco, Cambridge, London, Amsterdam

Keiling A, Takahashi K (2011) Review of Pi2 models. Space Sci Rev 161:63-148

Kim K-H, Takahashi K, Lee DH, Sutcliffe PR, Yumoto K (2005) Pi2 pulsations associated with poleward boundary intensifications during the absence of substorms. J Geophys Res 110:A01217. doi:10.1029/2004JA010780

Kokubun S, Erickson KN, Fritz TA, McPherron RL (1989) Local time asymmetry of Pc 4-5 pulsations and associated particle modulations at synchronous orbit. J Geophys Res 94:6607-6625

Kwon H-J, Kim K-H, Jun C-W, Takahashi K, Lee D-H, Lee E, Jin H, Seon J, PY-D, J Hwang (2013) Low-latitude Pi2 pulsations during intervals of quiet geomagnetic conditions $(K p=1)$. J Geophys Res 118:6145-6153

Lotz S, Heilig B, Sutcliffe P (2015) A solar-wind-driven empirical model of Pc3 wave activity at a mid-latitude location. Ann Geophys 33:225-234

Manninen J, Kleimenova NG, Kozyreva OV, Ranta A, Kauristie K, äkinen SM, Kornilova TA (2008) Ground-based observations during the period between two strong November 2004 storms attributed to steady magnetospheric convection. J Geophys Res 113 A00A09

Matsuoka H, Takahashi K, Kokubun S, Yumoto K, Yamamoto T, Solovyev SI, Vershinin EF (1997) Phase and amplitude structure of Pc 3 magnetic pulsations as determined from multipoint observations. J Geophys Res 102:2391-2403

Mazur NG, Fedorov EN, Pilipenko VA (2010) MHD waveguides in space plasma. Plasma Phys Rep 36:653-670

Mazur VA, Leonovich AS (2006) ULF hydromagnetic oscillations with the discrete spectrum as eigenmodes of MHD resonator in the near-earth part of the plasma sheet. Ann Geophys 24:1639-1648

Mier-Jedrzejowicz WAC, Southwood DJ (1979) The east-west structure of midlatitude geomagnetic pulsations in the $8-25 \mathrm{mHz}$ band. Planet Space Sci 27:617-630

Pilipenko V, Fedorov E, Heilig B, Engebretson MJ (2008) Structure of ULF Pc3 waves at low altitudes. J Geophys Res 113:A11208
Pilipenko V, Yumoto K, Fedorov E, Yagova N (1999) Hydromagnetic spectroscopy of the magnetosphere with Pc3 geomagnetic pulsations at 210 meridian. Ann Geophys 17:53-65

Ponomarenko PV, Waters CL, St-Maurice J-P (2010) Upstream Pc3-4 waves: experimental evidence of propagation to the nightside plasmapause/ plasmatrough. Geophys Res Lett 37:L22102. doi:10.1029/2010GL045416

Posch JL, Engebretson MJ, Pilipenko VA, Hughes WJ, Russell CT, Lanzerotti LJ (2003) Characterizing the long-period ULF response to magnetic storms. J Geophys Res 108:1029

Rostoker G (1995) The frequency spectrum of Pi 2 micropulsation activity and its relationship to planetary magnetic activity. J Atm Terr Phys 57:1117-1128

Roy M, Rao DRK (1998) Frequency dependence of equatorial electrojet effect on geomagnetic micropulsations. Earth Planets Space 50(10):847-851. doi:10.1186/BF03352178

Saka O, Alperovich L (1998) Sunrise effect on dayside Pc pulsations at the dip equator. J Geophys Res 103:13779-13786

Sarma SVS, Sastry TS (1995) On the equatorial electrojet influence on geomagnetic pulsation amplitudes. J Atm Terr Phys 57:749-754

Shinohara M, Yumoto K, Hosen N, Yoshikawa A, Tachihara H, Saka O, Kitamura T-I, Trivedi NB, Da Costa JM, Schuch NJ (1998) Wave characteristics of geomagnetic pulsations across the dip equator. J Geophys Res 103:11745-11754

Singer HJ, Russell CT, Kivelson MG, Greenstadt EW, Olson JV (1977) Evidence for the control of Pc 3,4 magnetic pulsations by the solar wind velocity. Geophys Res Lett 4:377-379

Stening RJ (1995) What drives the equatorial electrojet. J Atm Terr Phys 57:1117-1128

Sutcliffe PR, Heilig B, Lotz S (2013) Spectral structure of Pc3-4 pulsations: possible signatures of cavity modes. Ann Geophys 31:725-743

Sutcliffe PR, Lyons LR (2002) Association between quiet-time Pi2 pulsations, poleward boundary intensifications, and plasma sheet particle fluxes. Geophys Res Lett 29:1293-1296

Takahashi K, Liou K, Yumoto K, Kitamura K, Nose M, Honary F (2005) Source of Pc4 pulsations observed on the nightside. J Geophys Res 110:A12207. doi :10.1029/2005JA011093

Takalo J, Timonen J, Koskinen H (1994) Properties of AE data and bicolored noise. J Geophys Res 99:13239-13249

Troitskaya VA (1988) B. O. V, Diagnostics of the magnetosphere using multipoint measurements of ulf waves. Adv Space Res 8:413-425

Vellante M, Villante U, De Lauretis M, Barchi G (1995) Solar cycle variation of the dominant frequencies of $\mathrm{Pc} 3$ geomagnetic pulsations at $L=1.6$. Geophys Res Lett 23:1505-1508

Vero J, Luhr H, Vellante M, Best I, Strestik J, Miletits JC, Hollo L, Szendroi J, Zieger B (1998) Upstream waves and field line resonances: simultaneous presence and alternation in Pc3 pulsation events. Ann Geophys 16:34-48

Villante U, Lepidi S, Francia P, Vellante M, Meloni A, Lepping RP, Mariani F (1999) Pc3 pulsations during variable IMF conditions. Ann Geophys 17:490-496

Villante U, Tiberi P (2016) Occurrence and characteristics of nighttime ulf waves at low latitude. J Geophys Res Space Phys 121:4300-4315

Waters CL, Harrold BG, Menk FW, Samson JC, Fraser BJ (2000) Field line resonances and waveguide modes at low latitudes, 2, a model. J Geophys Res 105:7763-7774

Waters CL, Sciffer MD, Fraser BJ, Brand K, Foulkes K, Menk FW, Saka O, Yumoto K (2001) The phase structure of very low latitude ULF waves across dawn.J Geophys Res 106:15599-15607

Yagova N, Heilig B, Fedorov E (2015) Pc2-3 geomagnetic pulsations on the ground, in the ionosphere, and in the magnetosphere: MM100, CHAMP, and THEMIS observations. Ann Geophys 33:117-128

Yagova NV, Pilipenko VA, Baransky LN, Engebretson MJ (2010) Spatial distribution of spectral parameters of high latitude geomagnetic disturbances in the Pc5/Pi3 frequency range. Ann Geophys 28:1761-1775

Yagova N, Pilipenko V, Fedorov E, Vellante M, Yumoto K (1999) Influence of ionospheric conductivity on mid-latitude Pc3-4 pulsations. Earth Planets Space 51:129-138

Yumoto K (2001) The CPMN group, characteristics of Pi2 magnetic pulsations observed at the CPMN stations: a review of the STEP results. Earth Planets Space 53(10):981-992. doi:10.1186/BF03351695

Yumoto K, Saito T, Akasofu S-I, Tsurutani BT, Smith EJ (1985) Propagation mechanism of daytime Pc 3-4 pulsations observed at synchronous orbit and multiple ground-based stations. J Geophys Res 90:6439-6450 
Yumoto K, Tanaka Y, Oguti T, Shiokawa K, Yoshimura Y, Isono A, Fraser BJ, Menk FW, Lynn JW, Seto M, MM210 Obs. Group (1992) Globally coordinated magnetic observations along 210 magnetic meridian during STEP period: 1. preliminary results of low-latitude PC3's. J Geomag Geoelectr 44:61-276
Yumoto K, the MAGDAS Group (2006) MAGDAS project and its application for space weather. In: Gopalswamy N, Bhattacharyya A (eds) Solar influence on the heliosphere and earth's environment: recent progress and prospects. ISBN-81-87099-40-2, pp 309-405

\section{Submit your manuscript to a SpringerOpen ${ }^{\circ}$ journal and benefit from:}

- Convenient online submission

\section{- Rigorous peer review}

- Immediate publication on acceptance

- Open access: articles freely available online

- High visibility within the field

- Retaining the copyright to your article 